\title{
InAs Quantum Dot Formation Studied at the Atomic Scale by Cross-sectional Scanning Tunnelling Microscopy
}

\author{
J.M. Ulloa, P. Offermans and P.M. Koenraad \\ Department of Applied Physics, Eindhoven University of Technology, The Netherlands
}

\subsection{Introduction}

\subsubsection{Quantum dot formation}

Self-assembled quantum dots (QDs) have attracted much attention in the last years [1, 2]. These nanostructures are very interesting from a scientific point of view because they form nearly ideal zero-dimensional systems in which quantum confinement effects become very important. These unique properties also make them very interesting from a technological point of view. For example, InAs QDs are employed in QD lasers [3, 4], single electron transistors [5]. midinfrared detectors [6,7], single-photon sources [8,9], etc. InAs QDs are commonly created by the Stranski-Krastanov growth mode when InAs is deposited on a substrate with a bigger lattice constant, like GaAs or InP [10]. Above a certain critical thickness of InAs, three-dimensional islands are spontaneously formed on top of a wetting layer (WL) to reduce the strain energy. Once created, the $\mathrm{QDs}$ are subsequently capped, a step which is required for any device application.

For any application based on InAs QDs, an accurate control of their electronic properties is required. The electron and hole states in the dot are very sensitive to the QD size, shape and composition (as well as to the surrounding material), and therefore an accurate control of the QD structural properties is necessary for applications. This explains the strong effort dedicated in the last years to the structural characterization of QDs [11]. which is essential in order to have a deep understanding of the QD formation process. Although a lot of effort has been dedicated to study surface QDs, there are relatively few studies focused on the effect of the capping process [12-20]. Some of these studies have already shown significant differences in size, shape and composition between uncapped and capped ODs. For example, an important collapse of the OD height has been reported for InAs/GaAs ODs capped with GaAs [14, 15, 17-19], revealing the big influence of the capping process on the structural properties of the QDs. Indeed, critical issues affecting the dot take place during capping like dot decomposition, intermixing, segregation, As/P exchange and composition modulation in the capping layer. This means that the real buried QDs must be studied in order to understand the complete QD formation process. Cross-sectional scanning tunnelling microscopy is an especially useful technique for this purpose, because it allows the structure of the capped dots at the atomic scale to be assessed.

\subsubsection{Cross-sectional scanning tunnelling microscopy (X-STM)}

The scanning tunnelling microscope is part of the family of scanning probe microscopes, which are able to provide direct real space information of a physical property at the atomic scale. This 
is done by scanning a probe across the surface of a sample while recording the measured signal, which results in an image of the surface. In the case of scanning tunnelling microscopy (STM), invented in 1981 by Binnig and Rohrer [21], the probe consists of a metallic tip which is brought into tunnelling contact with a (semi-)conducting surface. The quantum mechanical tunnelling effect allows the electron to tunnel through the vacuum barrier even when the energy of the electrons is lower than the potential barrier (Fig. 5.1a). Since the tunnelling current depends exponentially on the distance between tip and sample, an atomic-scale height map of the surface density of states and topography can be acquired by adjusting the tip sample distance during scanning, using a feedback loop that keeps the tunnelling current constant (Fig. 5.1b).

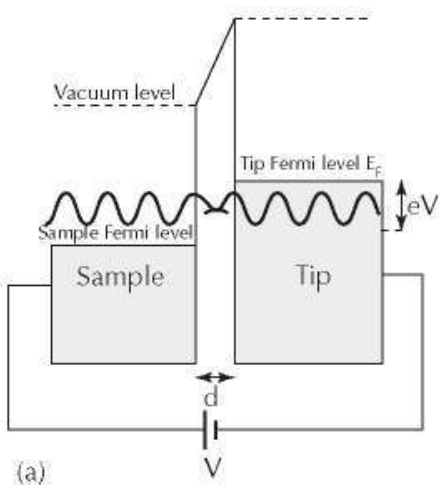

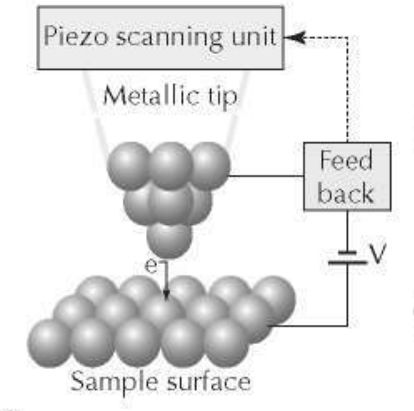

(b)

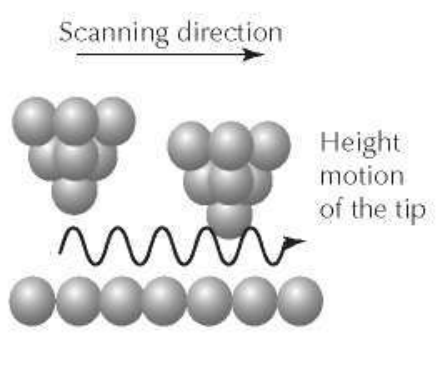

Figure 5.1 (a) Schematic showing the overlap and decay of tip and sample wave functions in the vacuum barrier: (b) Schematic of a scanning tunnelling microscopy with feedback loop.

In cross-sectional scanning tunnelling microscopy (X-STM), the direct visualization of nanostructures, which are generally embedded in several layers of semiconducting material, is possible by cleaving the sample and thereby exposing the cross-sectional surface of the buried nanostructures. The semiconductor wafers are cleaved along a natural cleavage plane to expose a crosssection of the epitaxial layers grown on the wafer. In the case of III/V semiconductors, the (110) and $(1 \overline{1} 0)$ cleavage planes of the zinc-blende crystal show a $1 \times 1$ surface unit cell reconstruction where the group III elements move into the surface while the group V elements move outward (Fig. 5.2). This buckling behaviour causes the energetic position of the dangling bond surface states of the type III and type V elements to move above and below the semiconductor band gap in the bulk, respectively. Therefore, depending on the polarity of the applied bias between tip and sample, either the empty states of the type III elements (Vsample $>0$ ) or the filled states of the type $V$ elements (Vsample $<0)$ are imaged (Fig. 5.3). Since the $(110)$ and $(1 \overline{1} 0)$ surface planes contain only half of the (001) layers within the zinc-blende crystal, the atomic rows observed in the cross-sectional STM image are separated by a bilayer distance in the growth [001] direction, which is equal to the lattice constant $a_{0}$. Because there are no surface states present in the band gap of most cleaved III/V semiconductors, the Fermi level at the cleaved surface is unpinned, i.e. it is not forced to assume a specific value in the band gap independent of its value in the bulk material. This allows the electronic properties of the bulk to be probed at energies near the band gap. The bulk states generally contribute with an electronic contrast to STM images that is not related to the actual physical topography of the surface, but governed by factors such as variations in the band gap, doping level and electron affinity.

The electronic contribution to the apparent height in the STM image can be suppressed to a large extent by scanning at high voltages $[22,23]$. Since the tunnelling probability decays exponentially with the height of the effective tunnelling barrier, the states with the highest energy contribute most to the tunnelling current. If a sufficiently large voltage is applied during empty state imaging of a heterostructure, the states contributing most to the tunnelling current have 

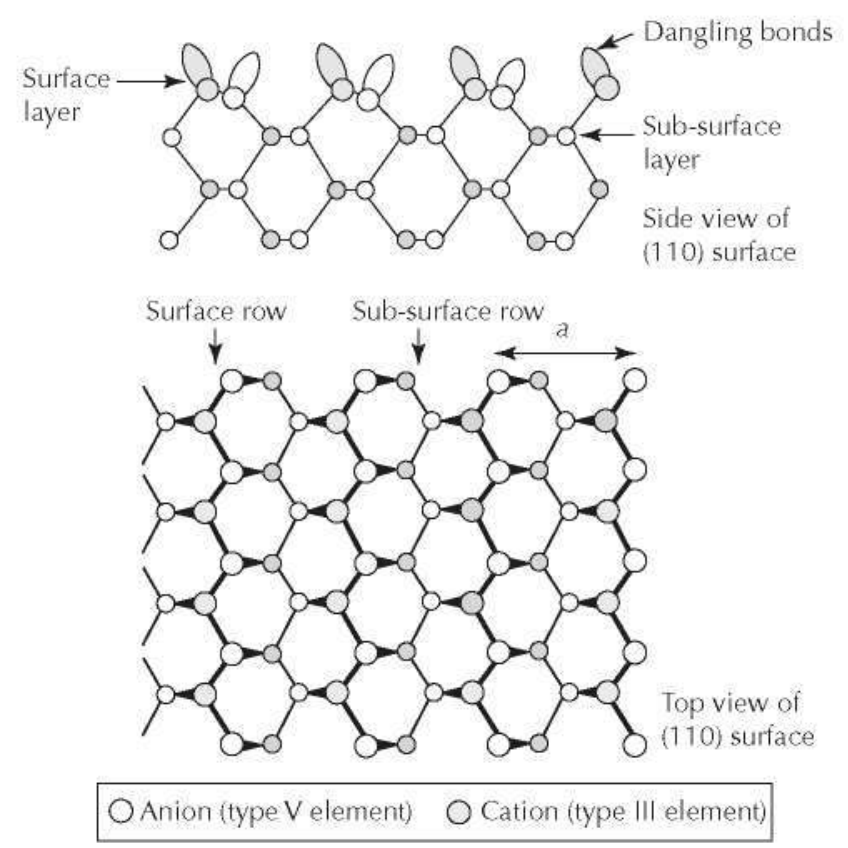

Figure 5.2 Schematic top and side view of $11 \mathrm{H} / \mathrm{V}$ semiconductor (110) surface.
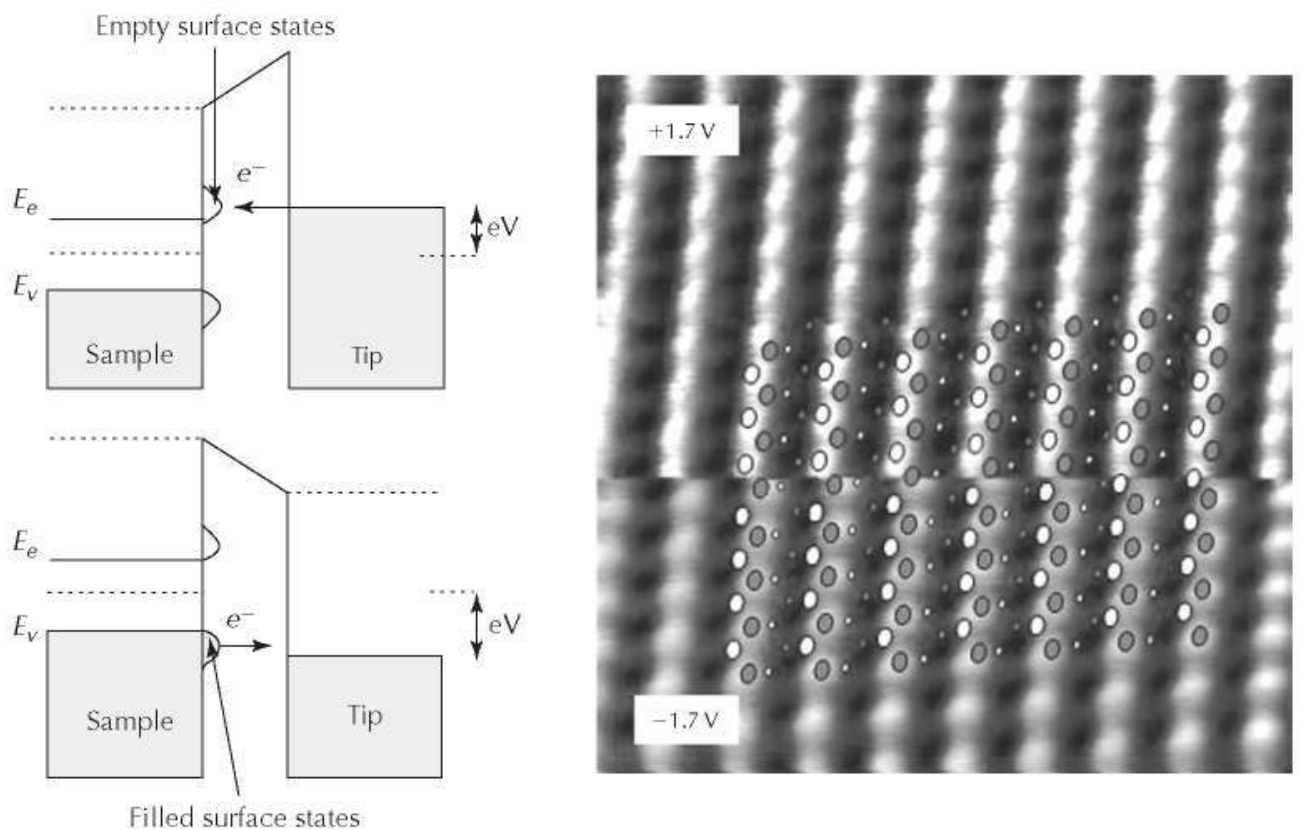

Figure 5.3 Schematic of tumnelling into empty and filled surface states, and STM image showing the atomic corrugation at both polarities.

energies far above the conduction band edge and the contribution of the lower lying states is negligible. Similarly, the voltage dependent sensitivity of the tunnelling current on doping level can be overcome by injecting electrons above the depletion region. In the case of filled states tunnelling (electron tunnelling from sample to tip), the valence band offset in a heterostructure is 
smaller with respect to the total barrier height than the conduction band offset, since the effective barrier height for tunnelling is increased by the band gap. Therefore, the resulting electronic contrast is smaller than in the case of empty states tunnelling (electron tunnelling from tip to sample). Thus, as demonstrated in $[22,23]$, at high (preferably negative) sample voltages, the electronic contribution to the height contrast is minimized, enabling the acquisition of the true (filled states) topography of the structure, such as the outward relaxation of the cleaved surface.

\subsubsection{X-STM topography and strain analysis}

When two materials with a different lattice constant are used in a heterostructure, it will accumulate strain. Stranski-Krastanov grown InAs quantum dots are the ultimate example of such strained heterostructures, but also (In,Ga) As quantum wells have built-in strain. When such a structure is cleaved, it reduces its built-in tensile or compressive strain by deforming the cleaved surface. Regions under compressive strain bulge outward while tensile strain depresses the cleaved surface (Fig. 5.4). The strain can be measured in two ways with cross-sectional scanning tunnelling microscopy:

- Strain in the plane of the surface can be deduced from the lattice spacing.

- Distortion normal to the surface can be measured using (filled states) topography imaging, as described before.

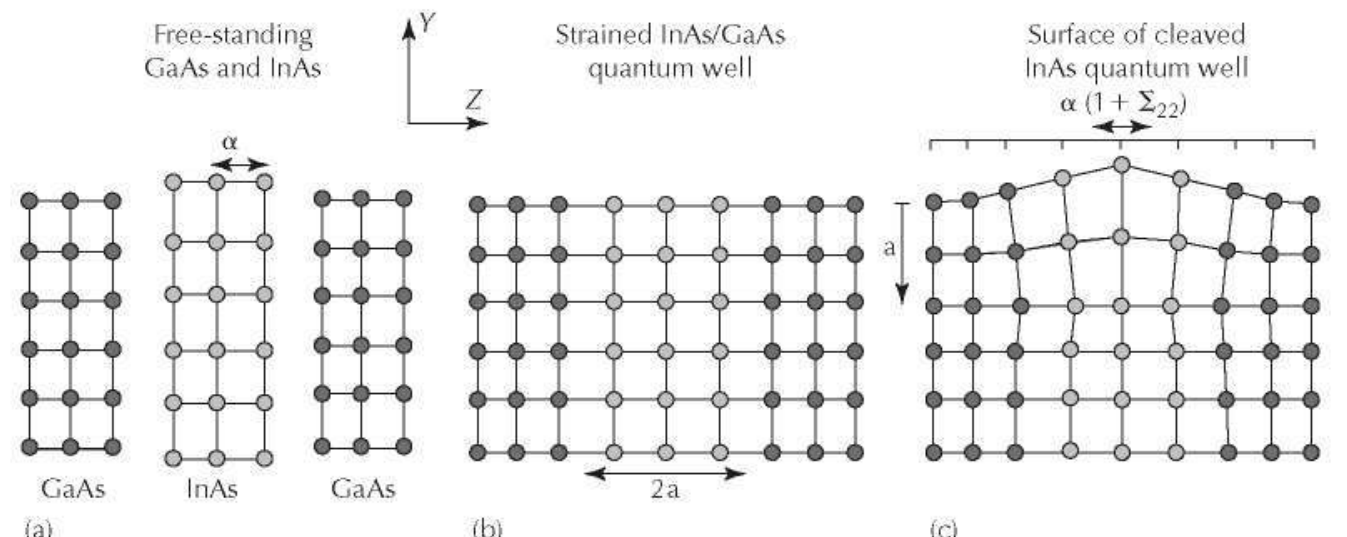

(a)

(b)

Figure 5.4 Strain relaxation at the cleaved surface of a strained quantum well. (a) Natural lattices of freestanding GaAs and InAs, (b) Due to lattice mismatch the InAs well is strained in GaAs. (c) The strain is released at the cleaved surface by outward relaxation.

The measured outward displacement and strain (measured by the change in lattice spacing) can be used to determine the indium composition of a strained (In,Ga) As nanostructure, by comparing the experimental data with the calculated relaxation and strain using elasticity theory [24]. In the case of quantum wells with a low concentration of indium $(<30 \%)$, or wetting layers, the indium distribution can also be obtained directly by counting the indium atoms, as will be shown in the next sections. However, in the case of InAs quantum dots the indium concentration is too high to distinguish individual indium atoms, and the only way to determine its composition is by fitting to the measured outward relaxation or change in lattice constant. To calculate the outward relaxation of a cleaved QD we use the package ABAQUS. It is based on continuum elasticity theory and performs a finite element calculation to solve the 3D problem, in which an isotropic material is considered. The measured size and shape of the QDs is introduced in the model and the composition is varied until the calculated profile fits to the experimental one. The real size and shape of the QDs can only be known after scanning a large number of dots, since they will be cleaved at a random position. Figure 5.5 shows the calculated outward relaxation 

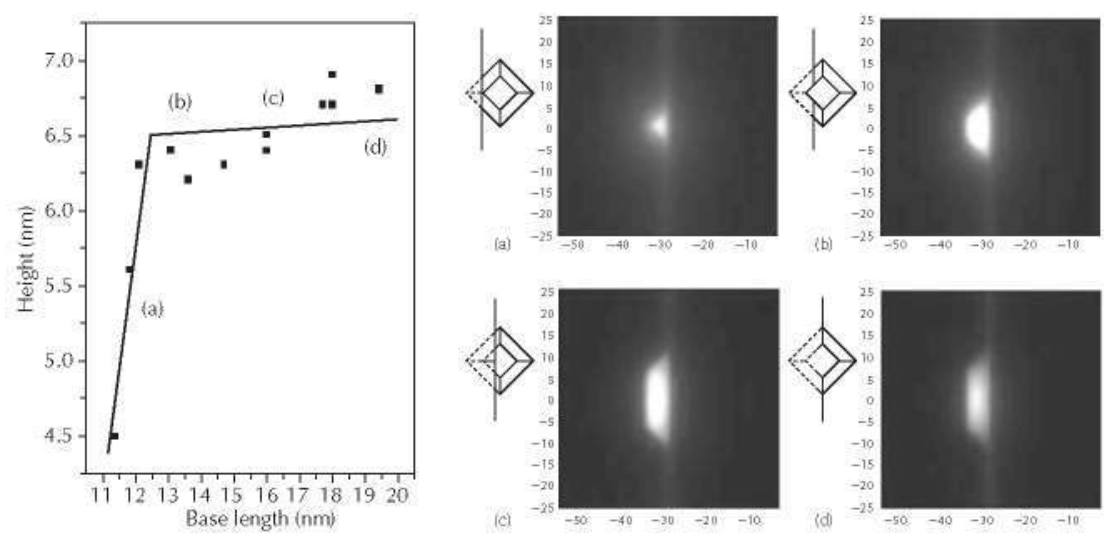

Figure 5.5 Expected (solid line) and measured by X-STM (squares) size distribution of cleaved InAs/GaAs QDs. The different sizes correspond to dots cleaved in different positions, as shown by the calculated outward relaxation for different cleavage planes.

of the surface of a cleaved InAs/GaAs QD and WL as a function of the position of the cleavage plane. The dots are considered to be truncated pyramids. The corresponding expected size distribution is shown by the solid line in the graph, in which the results of a real X-STM measurement are also shown (squares). Only after scanning a large number of cleaved dots can the maximum base length of the cross-section of the dot be determined, which is indicative for a cleavage near the dot centre. Only the outward relaxation profile of the dots cleaved close to the centre (dots in the region (d)) are used to compare with the model.

\subsubsection{Experimental set-up}

\subsection{Sample and tip preparation}

Small rectangular pieces (about $3.5 \times 10 \mathrm{~mm}^{2}$ ) are cleaved from a wafer containing the semiconductor heterostructure of interest. These wafers are normally 350-550 $\mu \mathrm{m}$ thick and are polished down with aluminum oxide powder to a thickness of about $100 \mu \mathrm{m}$, for easy cleavage. For an STM measurement it is essential to have good electric contact with the sample. Therefore, metallic contacts are evaporated at the top surface of the sample after treating it with an N2/ $\mathrm{H} 2$ plasma. A small scratch of about $0.5-1.5 \mathrm{~mm}$, which extends to a small notch at the side of the sample, is made at the top surface of the sample using a diamond pen. This scratch facilitates the cleavage of the sample and provides a fixed starting point for the propagation of the cleavage plane. The sample is clamped on a sample holder between two metal bars that can be screwed together, as shown in Fig. 5.6. Only one corner of the sample is clamped for the unconstrained propagation of the cleavage plane. Between the metal bars and the two surfaces of the sample, at the position where the sample is to be clamped, thin slices of indium are placed. The sample holder is heated in order to melt the indium before tightening the screws. The indium provides an even pressure distribution on the sample, preventing it from cleaving during tightening of the screws and slipping out of the holder when the sample is degassed subsequently in the UHV system. The sample is cleaved in the STM chamber using a gentle touch by a manipulator (the so-called "wobble stick") just before the measurement as shown in Fig. 5.6. A characteristic crack pattern is also shown in Fig. 5.6 [25]. After cleavage, the sample is placed into the sample stage and moved towards a mounted tip, with the help of a CCD camera equipped with a telelens. First, the sample is brought close to the tip and then further approached until tunnelling contact is done automatically by the system. After the first tunnelling contact, the tip is retracted and moved several $100 \mathrm{~nm}$ towards the edge of the cleavage surface where the grown layers (epilayers) are located. The process of making tunnelling contact and moving towards the epilayers is repeated until the tip moves over the edge of the sample. At this point tunnelling contact is not achieved, which indicates that the location of the epilayers is within several $100 \mathrm{~nm}$. 


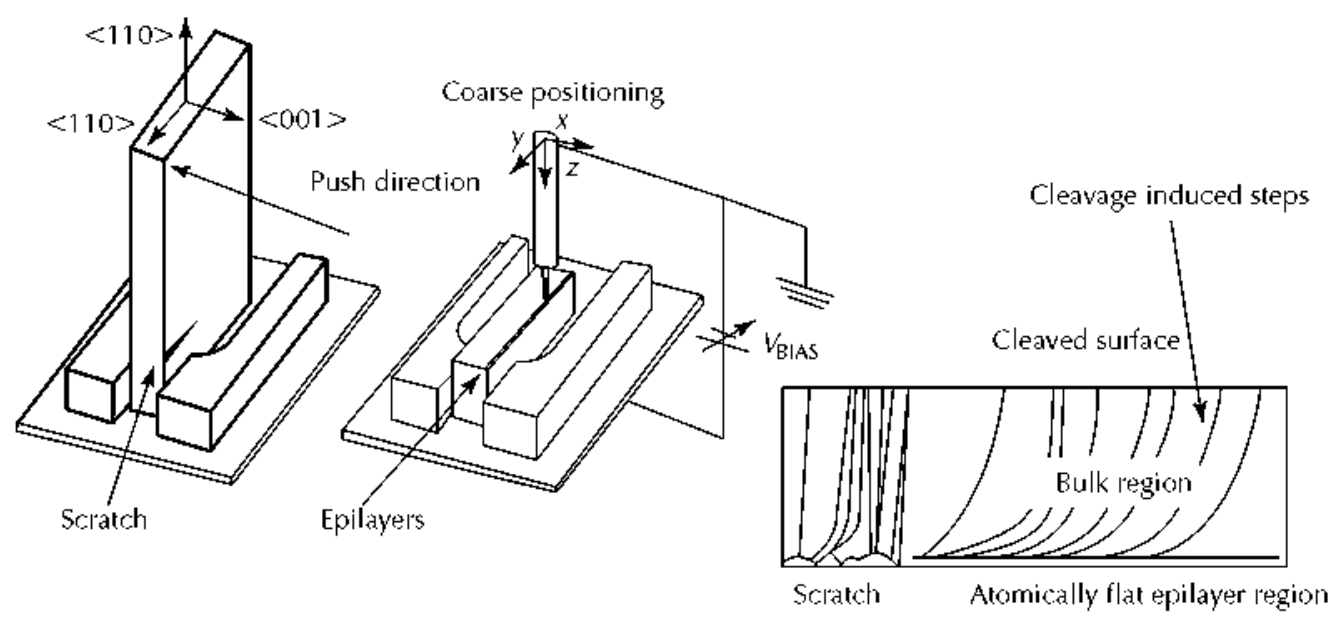

Figure 5.6 Schematic view of the sample cleavage process.

The tips are made of $99.97 \%$ pure polycrystalline tungsten wire with a diameter of $0.25 \mathrm{~mm}$. A short piece of this wire $(-5 \mathrm{~mm})$ is spot welded onto an Omicron tip holder and cleaned for use in UHV. The tips are then electrochemically etched with a 2.0 molar potassium hydroxide solution. The top $1-1.5 \mathrm{~mm}$ of the tip is put into the solution and a positive voltage $(4-5 \mathrm{~V})$ is applied to the tip wire. A platinum-iridium $(90 \% / 10 \%)$ spiral serves as a counter-electrode. As the reaction products dissolve in the etchant, they sink down along the wire, which is visible from the local change of the diffractive index of the solution. Because of the geometry, the reaction velocity is the highest at the point where the tungsten wire penetrates the surface of the solution. This causes necking of the wire at the surface of the etching solution, as the reaction products owing down the tip shield the rest of the wire. Eventually, the wire will break at the neck leaving a very sharp tip. A current limiter is used to interrupt the etching process immediately ( $<1 \mu$.s) alter the breaking of the wire. The tips are degassed after loading into the UHV system and treated with a $700 \mathrm{eV}$ argon ion bombardment in order to mechanically stabilize the tip.

5.1.2.2.2 The STM unit and the UHV system

A commercially available room-temperature STM unit (Omicron STM-1 TS2) is used. The STM set-up is very sensitive to external vibrations, as the tip-sample distance during tunnelling is only a few angstrom. Moreover, the apparent height of the atomic corrugation visible in the STM images is only $20 \mathrm{pm}$. Therefore, the set-up is vibration isolated with different damping systems. The scanner unit is suspended on a set of springs and stabilized by an eddy current damping system. The eddy current damping system consists of copper fins that surround the scanner and are placed between permanent magnets when the scanner unit is in its suspended position. The STM unit, together with the rest of the set-up. is mounted on a heavy metal tabletop. Rubber dampers between this tabletop and the supporting frame filter out high-frequency vibrations. Lowfrequency vibrations are suppressed by four active damping elements that are mounted between the floor and the frame. In this active damping system several motion detectors are present and several actuator coils counteract the detected motion of the system. Finally the entire set-up is standing on a heavy concrete platiorm that is decoupled from the building.

A factor of critical importance in cross-sectional scanning tunnelling microscopy on III/V semiconductors is the production of an atomically flat cross-sectional surface that is free of contamination/oxidation, in order to obtain an electronically unpinned surface. Therefore, cleavage of the samples and the subsequent measurement are performed in a home-built ultra-high vacuum set-up (Fig. 5.7). The central vacuum chamber (STM), in which the STM unit is positioned, is pumped down by an ion-getter pump (IGP) with a titanium sublimation element (TSE) to a pressure lower than $5 \times 10^{-11}$ torr (Varian VacIon Plus, 300 litre/sec). During X-STM 


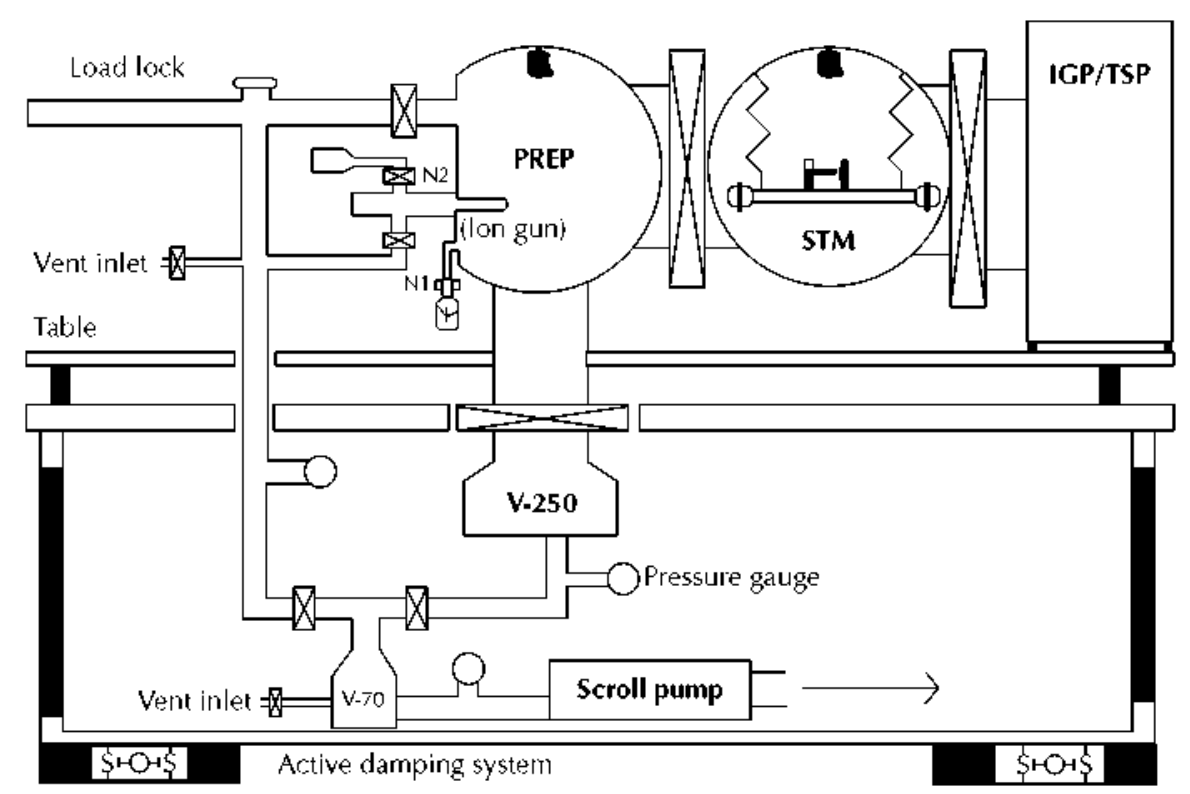

Figure 5.7 Schematic drawing of the UHV system containing the STM and damping system.

measurements the inner panel of the IGP/TSE can be cooled down with liquid nitrogen, which enhances the pump speed by a factor of two. In the preparation chamber (PREP), which is separated by a valve from the STM chamber, the tips and samples can be degassed with a baking unit and tips can be treated with an argon ion bombardment. Two oil-free turbo-molecular pumps (Varian $\mathrm{V}-250$ and $\mathrm{V}-70$ ), installed in series, keep the preparation chamber at a pressure of approximately $6 \times 10^{-10}$ torr. The preparation chamber is connected to a load lock, for loading and unloading the tips and samples without seriously affecting the pressure in the preparation and STM chamber. The load lock is pumped with one of the turbo-molecular pumps (V-70) to a pressure of about $10^{-6}$ torr. An XDS5 dry scroll pump provides the necessary pre-vacuum of about $10^{-1}$ torr. During the $\mathrm{X}$-STM measurements all pumps are switched off to prevent vibrations, except the IGP/TSE as this pump contains no moving parts. Two wobble sticks in the STM and preparation chamber are used for manipulation and transportation of the tips and samples.

\subsection{Formation of the wetting layer}

The formation of InAs wetting layers (WLs) has attracted relatively little attention compared to quantum dot (OD) formation [26-28]. In the simple picture of Stranski-Krastanov growth. after the build-up of a critical amount of strain, 2D layer growth is followed by $\mathrm{QD}$ formation. It has become increasingly clear, however, that such a simple picture is far from reality. Recently, In incorporation during pseudomorphic InAs/GaAs growth and QD formation was observed by in situ stress measurements [29].

In this section we analyse the composition and segregation of InAs WLs by either directly counting the indium atoms or by analysis of the outward displacement of the cleaved surface as measured by X-STM.

As mentioned in the introduction, the measured outward relaxation profile at high negative voltage can be compared to the calculated one to deduce the In concentration in the WL. By modelling the indium segregation, the outward displacement of the segregated WL can be calculated by integration of the analytical expression derived by Davies for the outward displacement of a cleaved quantum well [24]. Several models for indium segregation have been proposed 
[30-33]. We use the phenomenological model of Muraki et al. [31], which has been shown to describe well the indium composition $x(n)$ of InAs WLs [28]:

$$
x(n)=\left\{\begin{array}{cc}
0, & n<1 \\
\left(1-R^{n}\right), & 1 \leq n \leq N \\
\left(1-R^{N}\right) R^{n-N}, & n>N
\end{array}\right.
$$

where $n$ is the monolayer (ML) index, $N$ is the total amount of deposited indium and $R$ is the indium segregation coefficient. $N$ and $R$ are determined by fitting the calculated relaxation profile to the measured relaxation profile.

The WLs were grown by molecular-beam epitaxy (MBE) on doped GaAs (100) wafers. In sample A, three different sets of WLs were grown at $495^{\circ} \mathrm{C}$ by deposition of $1.5 \mathrm{ML}, 2.0 \mathrm{ML}$ and $2.5 \mathrm{ML}$ of InAs, respectively, at a growth rate of $0.1 \mathrm{ML} / \mathrm{s}$. Each layer was repeated two times, separated by a $50 \mathrm{~nm} \mathrm{GaAs}$ buffer layer, also grown at $495^{\circ} \mathrm{C}$. A growth interruption of 10 seconds has been applied after the growth of each layer. No dot formation was observed for the layers with $1.5 \mathrm{ML}$ indium deposition. In sample B, two sets of WLs were grown at $480^{\circ} \mathrm{C}$ by deposition of $2.0 \mathrm{ML}$ of InAs at a high and a low growth rate of $0.1 \mathrm{ML} / \mathrm{s}$ and $0.01 \mathrm{ML} / \mathrm{s}$, respectively. Each layer was repeated two times and capped by a $20 \mathrm{~nm}$ GaAs layer grown at $480^{\circ} \mathrm{C}$, followed by a $30 \mathrm{~nm}$ GaAs layer grown at $580^{\circ} \mathrm{C}$. A growth interruption of 10 seconds has been applied after the growth of each layer. In sample C, one set of InAs layers was grown in GaAs while a second set was grown in AlAs barriers. The InAs layers were grown at $500^{\circ} \mathrm{C}$ by deposition of $1.9 \mathrm{ML}$ of InAs in a cycled way, i.e. with a 3 second pause after each deposition of $0.25 \mathrm{ML}$, at a growth rate of $0.043 \mathrm{ML} / \mathrm{s}$. The following layer sequence was used: $20 \mathrm{~nm}$ GaAs $/ 1.9 \mathrm{ML} \mathrm{InAs} / 40 \mathrm{~nm}$ GaAs/1.9 ML InAs/40 nm GaAs/50 nm GaAs (doped $\left.1 \times 10^{18} \mathrm{~cm}^{-2}\right) / 20 \mathrm{~nm} \mathrm{GaAs} / 4 \times(20 \mathrm{~nm}$ AlAs/1.9 ML InAs $/ 20 \mathrm{~nm} \mathrm{AlAs/40} \mathrm{nm} \mathrm{GaAs).} \mathrm{To} \mathrm{reduce} \mathrm{interface} \mathrm{roughness,} \mathrm{the} \mathrm{bottom} \mathrm{AlAs}$ barriers were grown at $600^{\circ} \mathrm{C}$ followed by a growth interruption prior to InAs deposition.

Sample A was used to study the effect of the amount of indium deposition on the WL formation. We measured the relaxation profiles of the WLs and fitted these with calculated relaxation profiles, by adjusting the fit parameters $N$ and $R$. The resulting segregation profiles were verified by counting directly the number of indium atoms in the WL as a function of distance in growth direction. For the counting procedure, we selected four high-quality images of each layer, such as the one shown in Fig. 5.8. The relaxation and segregation profiles are shown in Fig. 5.9.

(a)

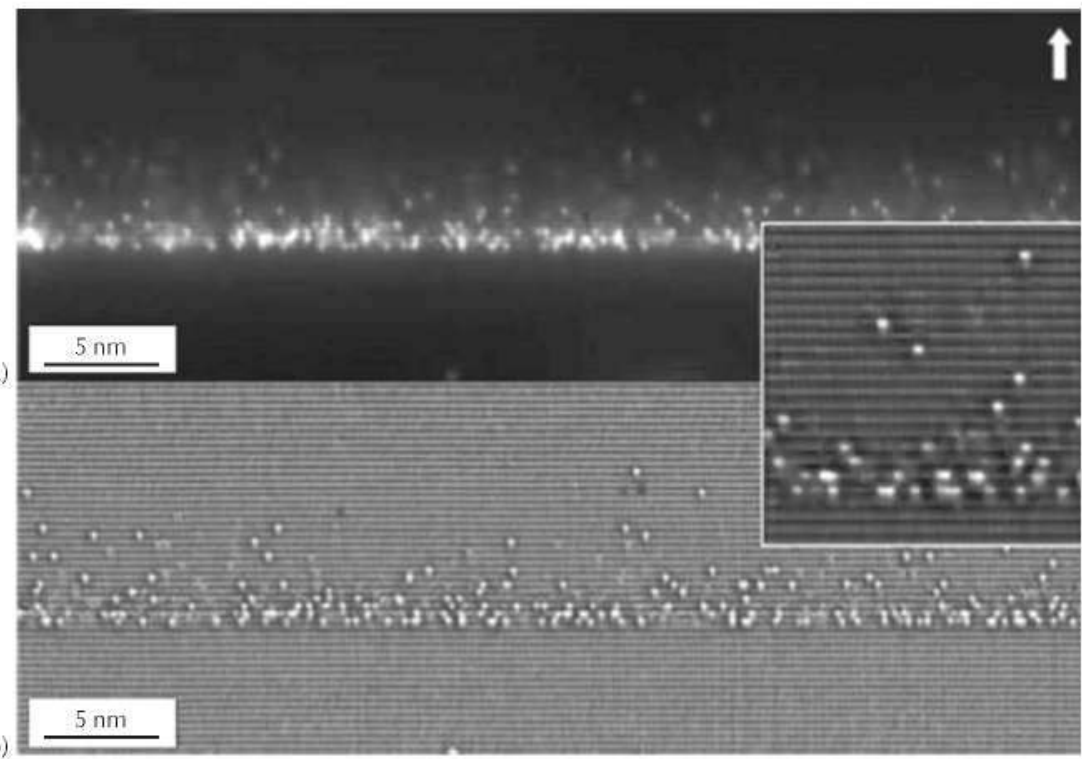

Figure 5.8 (a) Empty states X-STM image of a segregated 2.OML InAs WL. The arrow indicates the growth direction. (b) The same image treated with a high-pass Fourier filter. The inset shows an enlarged view of part of the image. 

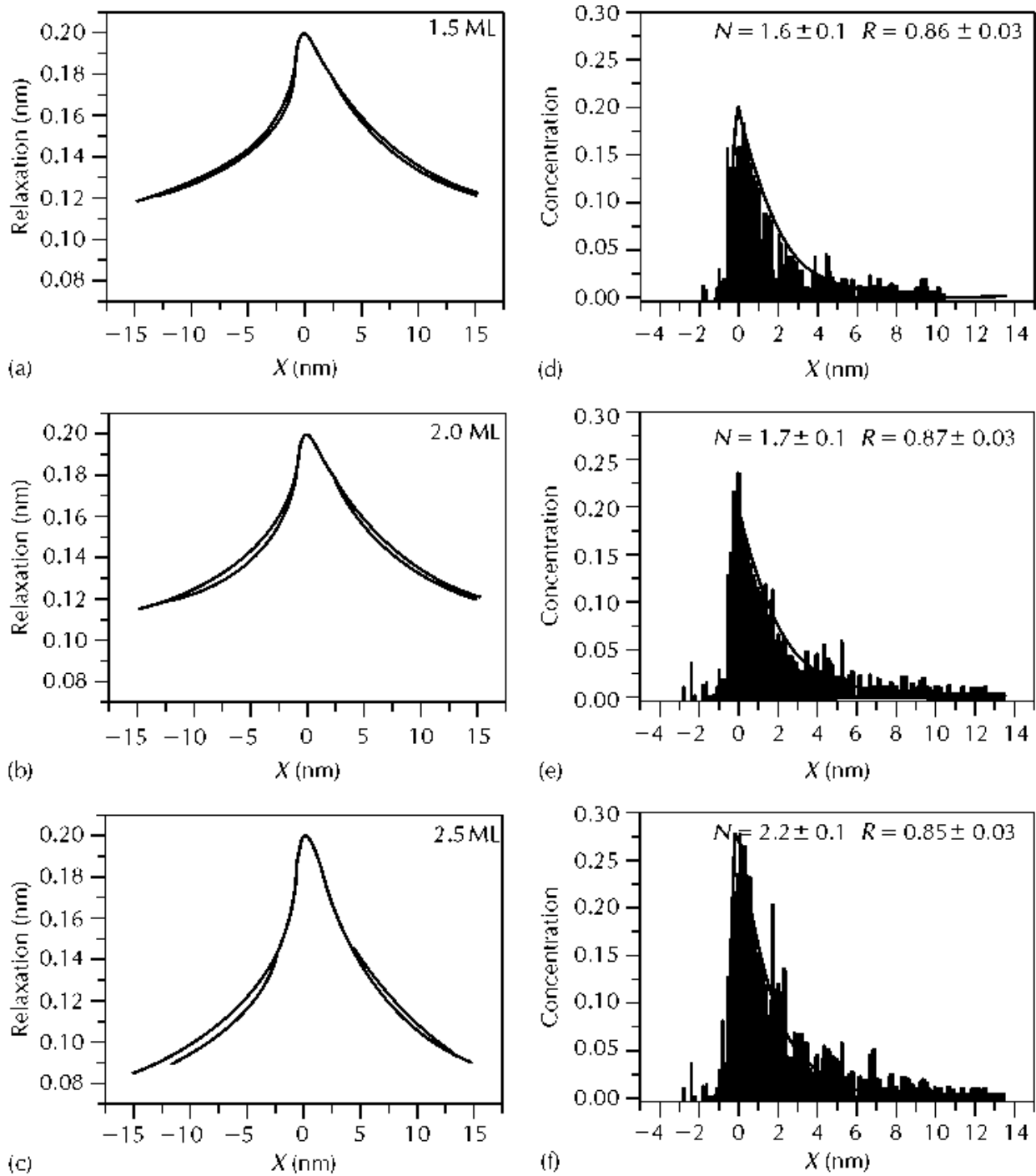

Figure 5.9 Measured and fitted relaxation profiles of the $1.5 \mathrm{ML}$ (a). $2.0 \mathrm{ML}$ (b) and $2.5 \mathrm{ML}$ (c) InAs WLs of sample. A. The black curves in (d), (e) and (f) show the segregation profiles corresponding to the fitted relaxation profiles in (a), (b) and (c). The columns indicate the counted indium concentration in the WL as a function of distance. $\mathrm{X}$ in the growth direction.

In all three cases, we find an excellent agreement between the indium profile determined from the outward relaxation of the surface, and the direct counting procedure. For the $1.5 \mathrm{ML} W \mathrm{~L}$, the measured amount of indium $N$ corresponds to the deposited amount, since no indium has gone into dot formation. For the 2.0ML and 2.5 ML WLs, however, we find a clear indium enrichment of the WL, despite dot formation.

In Stranski-Krastanov growth mode, strain builds up until the critical amount of indium for dot formation is deposited [34]. It has been shown that only part of the deposited amount of indium contributes to the strain, by incorporation into the lattice, while the remaining indium forms a floating layer on the surface [29]. During dot formation, part of the floating indium is transferred by lateral mass transport to the dots. The amount of indium that remains in the dots. however, is strongly reduced by the capping process, which dissolves the top of the dots back into the WL, as we will show in sections 5.2 and 5.4. The dissolved indium adds to the remaining 
floating indium, and is eventually incorporated into the lattice, during continued capping. That is the reason for the observed indium enrichment of the WL after dot formation.

We used sample B to study the effect of a reduced growth rate on the WL formation. It is known that a reduced growth rate leads to an increased QD size and a reduced QD density. However, it is not a priori clear how this will affect the formation of the segregated WL in the buried structure. In Fig. 5.10 we show the average measured and calculated relaxation profiles of the InAs WLs of sample B. The dashed lines indicate the relaxation profiles calculated directly from indium atom counting using different images. We find that the high $(0.1 \mathrm{ML} / \mathrm{s})$ and low $(0.01 \mathrm{ML} / \mathrm{s})$ growth rate InAs WLs can be described by the same parameters within errors. However, there is, as expected, a marked difference in the size of the QDs, shown in Fig. 5.11. Whereas the QD grown at the high growth rate appears as a rather flat, disk-like shape with a height of $3 \mathrm{~nm}$ (Fig. 5.11a), the QDs grown at low rate show an indium distribution with a reversed truncated cone shape [35] with a height of $5.4 \mathrm{~nm}$ (Fig. 5.11b).
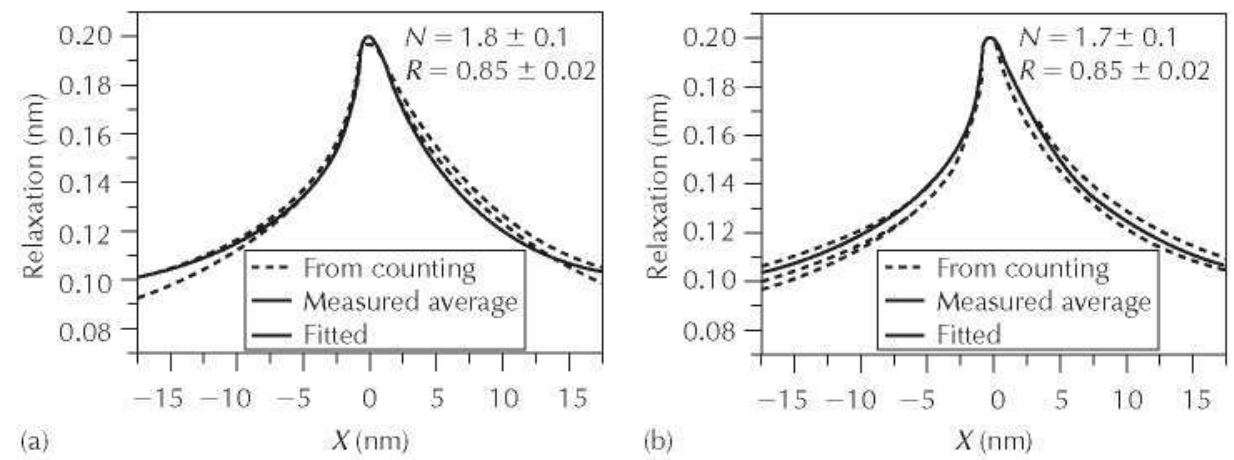

Figure 5.10 Measured and fitted relaxation profiles of the high (a) and low (b) growth rate InAs WLs of sample B. The dashed lines indicate the relaxation profiles calculated directly from counted segregation profiles.

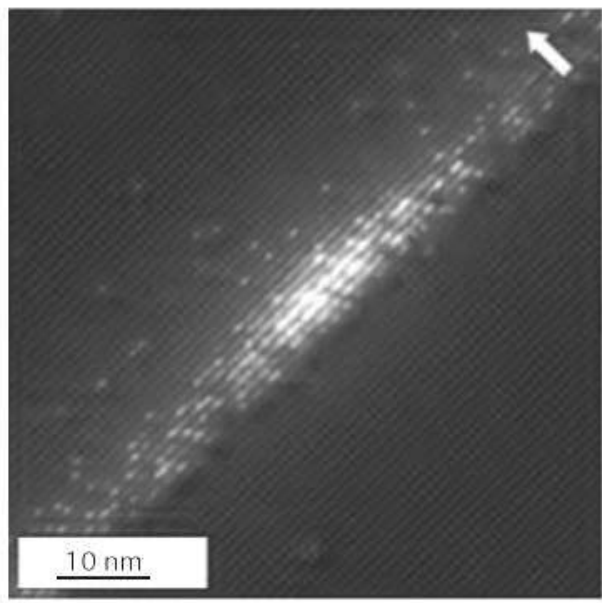

(a)

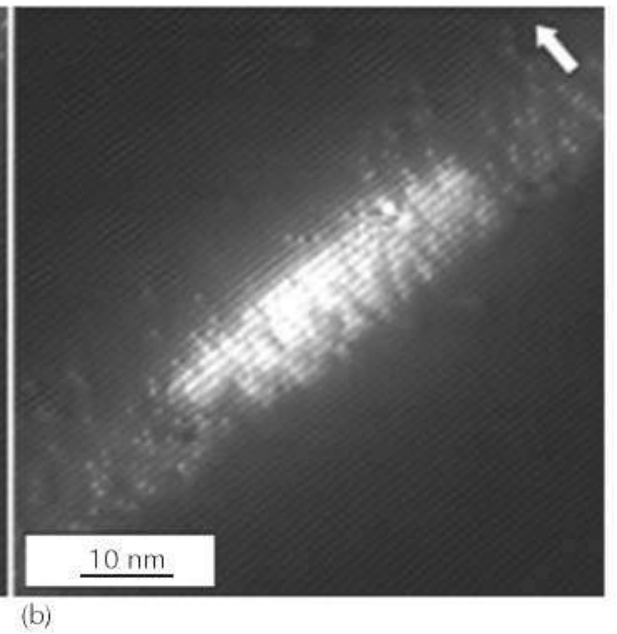

(b)

Figure 5.11 X-STM images of typical quantum dots found in the high (a) and low (b) growth rate WLs. The arrow indicates the growth direction.

We studied the effect of the host material by analysing the segregation of InAs WLs grown in the AlAs barriers of sample C, and comparing this to the segregation of InAs WLs grown, under the same growth conditions, in a GaAs matrix. It has been shown that QD formation in the InAs/AlAs system is kinetically limited due to a reduced lateral In migration on the AlAs surface, because of the larger Al-In bond strength [36]. In the next section the marked differences in the structural properties of the dots grown in GaAs and AlAs will be discussed [37]. Figure 5.12 
shows the averaged measured and fitted relaxation profiles. We find that the vertical indium segregation in $\mathrm{AlAs}$ and GaAs can be described by almost the same parameters, in agreement with [27]. This indicates that, in contrast to the lateral In migration, the vertical indium segregation is strain driven rather than determined by the chemical bond strength.
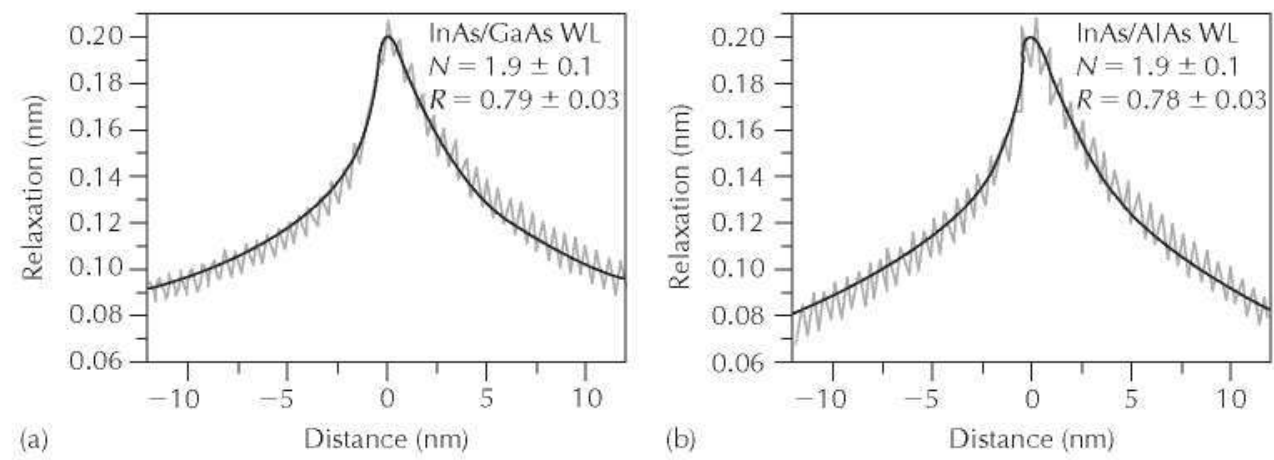

Figure 5.12 Measured and fitted relaxation profiles of the segregated InAs WLs in GaAs (a) and AlAs (b).

It is known that vertical indium segregation is reduced at lower growth temperatures [38]. As will be shown in section 5.4 .1 , we have reported that reducing the growth temperature to $300^{\circ} \mathrm{C}$ after capping of the WLs with three monolayers of GaAs leads to a dramatic reduction of the indium segregation [19]. However, such a capping procedure also leads to the almost complete dissolution of the QDs into the capping layer due to their partial coverage.

Another drastic example of the effects of partial capping is the formation of quantum rings (QRs). QRs can be grown by the partial capping of QDs with $2 \mathrm{~nm}$ of GaAs and subsequent annealing [39]. Recently, we observed that during this process, a second layer of indium accumulates on the surface of the capping layer, which is due to vertical segregation of indium from the WL and to lateral migration on the surface of indium atoms that have been expelled from the QDs during QR formation [40-43]. After continued capping, the second layer of indium itself forms a segregated indium distribution.

Finally, we show in Fig. 5.13 an overview image of the WLs of sample B. Surprisingly, one of the layers showed a shallow V-groove in which a QD was formed. The V-groove was unintentionally created on the GaAs substrate. It can clearly be seen that a large amount of indium atoms have accumulated in the V-groove. By comparing the extent of the indium segregation inside and

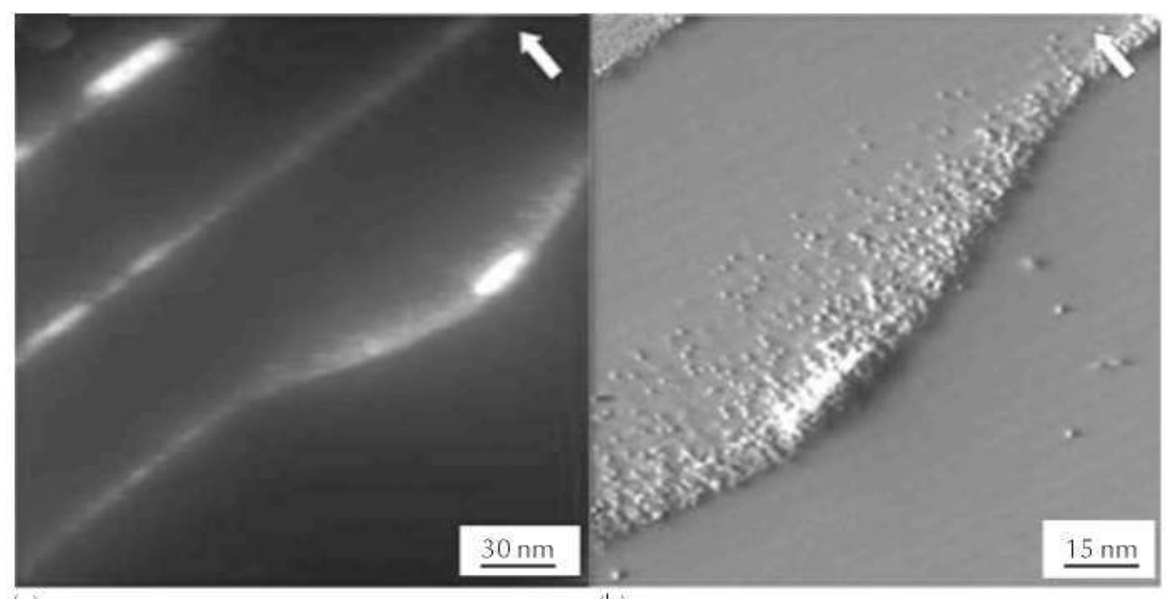

(a)

(b)

Figure 5.13 (a) X-STM overview image showing a shallow V-groove in one of the layers of sample B. (b) X-STM current image showing an enlarged view of the V-groove. The arrow indicates the growth direction. 
outside the V-groove, it can be seen that during GaAs overgrowth, the indium segregation and migration facilitates a rapid planarization of the growth front, in the presence of indium atom accumulation in the V-groove.

To summarize, we have shown that the composition of (segregated) InAs WLs can be determined by either directly counting the indium atoms or by analysis of the outward displacement of the cleaved surface as measured by X-STM. We used this approach to study the eflects of the deposited amount of indium, the InAs growth rate, and the host material on the formation of the WLs. We conclude that the formation of (segregated) WLs is a delicate interplay between surlace migration, strain-driven segregation and the dissolution of quantum dots during overgrowth.

\subsection{Dependence of the QD structural properties on the substrate material (GaAs vs AlAs)}

Self-assembled InAs quantum dots embedded in an AlAs matrix have been of recent interest because of their larger confinement potential compared to InAs QDs in a GaAs matrix and their use in resonant tunnelling devices [44]. In order to understand the formation and the electronic properties of InAs QDs in AlAs, detailed information about the composition of the QDs is needed. Several studies have been reported on the effect of indium supply and growth temperature on the structural and optical properties of InAs QDs grown on AlAs [45,46]. It has been shown that InAs QDs grown on AlAs exhibit smaller sizes and larger densities compared to InAs QDs that were grown on GaAs under similar growth conditions. This has been attributed to a reduced diffusion of In adatoms on the AlAs surface due to a higher surface roughness and the larger Al-In bond strength [45].

In this section we use X-STM measurements to determine the composition of InAs QDs and WLs grown on AlAs, and compare this to InAs ODs that were grown on GaAs under identical growth conditions.

The QDS were grown by MBE on doped GaAs (100) wafers. During growth of the QDs the substrate temperature was maintained at $500^{\circ} \mathrm{C}$. A $1.9 \pm 0.1$ monolayer (ML) of InAs was grown in a cycled way, i.e. with a 3 second pause after each deposition of $0.25 \mathrm{ML}$, at a slow growth rate of $0.043 \mathrm{ML} / \mathrm{s}$. Alter a $500 \mathrm{~nm}$ doped $\left(1 \times 10^{18} \mathrm{~cm}^{-2}\right)$ GaAs buller layer the following sequence was grown: $20 \mathrm{~nm}$ GaAs/1.9ML InAs/40 nm GaAs/1.9ML InAs/40 nm GaAs/50nm GaAs (doped $\left.1 \times 10^{18} \mathrm{~cm}^{-2}\right) / 20 \mathrm{~nm}$ GaAs/4 $\times(20 \mathrm{~nm}$ AlAs $/ 1.9 \mathrm{ML}$ InAs/20nm AlAs/40 nm GaAs). To reduce interface roughness, the bottom AlAs barriers were grown at $600^{\circ} \mathrm{C}$. followed by a growth interruption prior to InAs deposition.

Figure 5.14a shows a large-scale filled-states topography X-STM image of the structure. Three layers of ODs grown on AlAs and two layers of ODs grown on GaAs are visible in the image. Compared to GaAs, the ODs grown on AlAs show a smaller size and have a significantly larger density of about $3 \times 10^{11} \mathrm{~cm}^{-2}$. Many $\mathrm{ODs}$ were imaged and the largest ones selected for analysis. It can then be assumed that these QDs are cleaved near their middle.

In Fig. 5.14b and c we compare high-voltage filled-states topography images of individual QDs grown on GaAs and AlAs, respectively. These images show the surface relaxation which varies with the local indium distribution in the QDs. From the contrast in the images it can be seen that the InAs/AIAs QD has a more homogeneous surlace relaxation than the InAs/GaAs QD. We calculated the outward relaxation and the strain distribution of the QDs with the finite element calculation package ABAOUS. The OD shape was modelled by truncated pyramids with sizes determined by the X-STM measurements while the indium distribution was varied in order to get the optimal fit to the measured outward relaxation. The best results were obtained by allowing the modelled $\mathrm{ODs}$ to be cleaved at a plane $1 \mathrm{~nm}$ above their diagonal. The diagonal base length and the height of the InAs/GaAs $Q D$ are $28.4 \mathrm{~nm}$ and $6 \mathrm{~nm}$, respectively. For the InAs/AIAs QD the diagonal base length is $19 \mathrm{~nm}$ and the height is $4.2 \mathrm{~nm}$. The calculated relaxation of the cleaved surface of the QDs is shown in Fig. 5.14d and e using the same colour scale as in the corresponding X-STM images (Fig. 5.14b and c). From the calculated strain distribution, lattice constant profiles were derived which were used to verily the fitting results.

Figure 5.15 shows the measured and calculated outward relaxation profiles (a) and (b) and lattice constant profiles (c) and (d) taken in the growth direction through the centre of the QDs. 

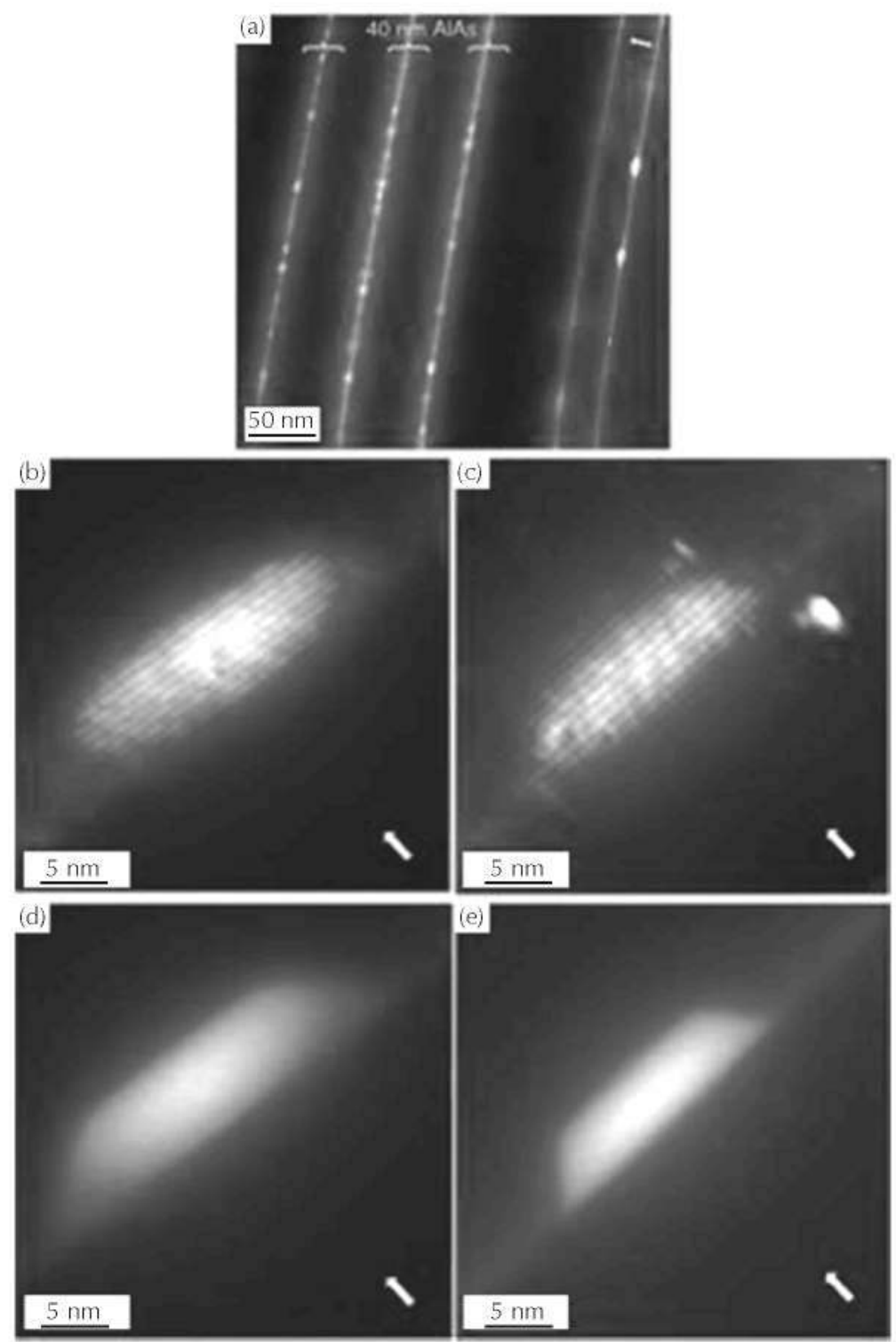

Figure 5.14 Filled states topography X-STM image of (a) three OD layers embedded in 40 nm thick AlAs barriers and two $Q D$ layers grown in GaAs, (b) InAs/GaAs QD. (c) InAs/AlAs $Q D$. (d) and (e) show the calculated outward relaxation corresponding to (b) and (c). The colour scale for (b) and (d) is $O$ (dark) to $600 \mathrm{pm}$ (bright). The colour scale for (c) and (e) is $O$ (dark) to $450 \mathrm{pm}$ (bright). $\mathrm{V}_{\text {sample }}=-3 \mathrm{~V}$.

From the change in lattice constant, which is determined by the strain distribution in and around the QDs, it can be seen that there is compressive strain above and below the QDs.

For the InAs/GaAs QD, there is a clear increase in lattice constant towards the top of the QD, which indicates an increasing indium concentration. This can also be seen by the slight asymmetry in the relaxation profile of the InAs/GaAs QD. From X-STM and photocurrent experiments, it has been shown that low-growth rate InAs/GaAs QDs have an increasing indium concentration in the growth direction $[47,48]$. However, other groups have reported InGaAs QDs with laterally non-uniform indium compositions showing an inverted-triangle, trumpet or truncated reversed-cone shape $[26,49-51]$. We find that the indium distribution of our low-growth rate 

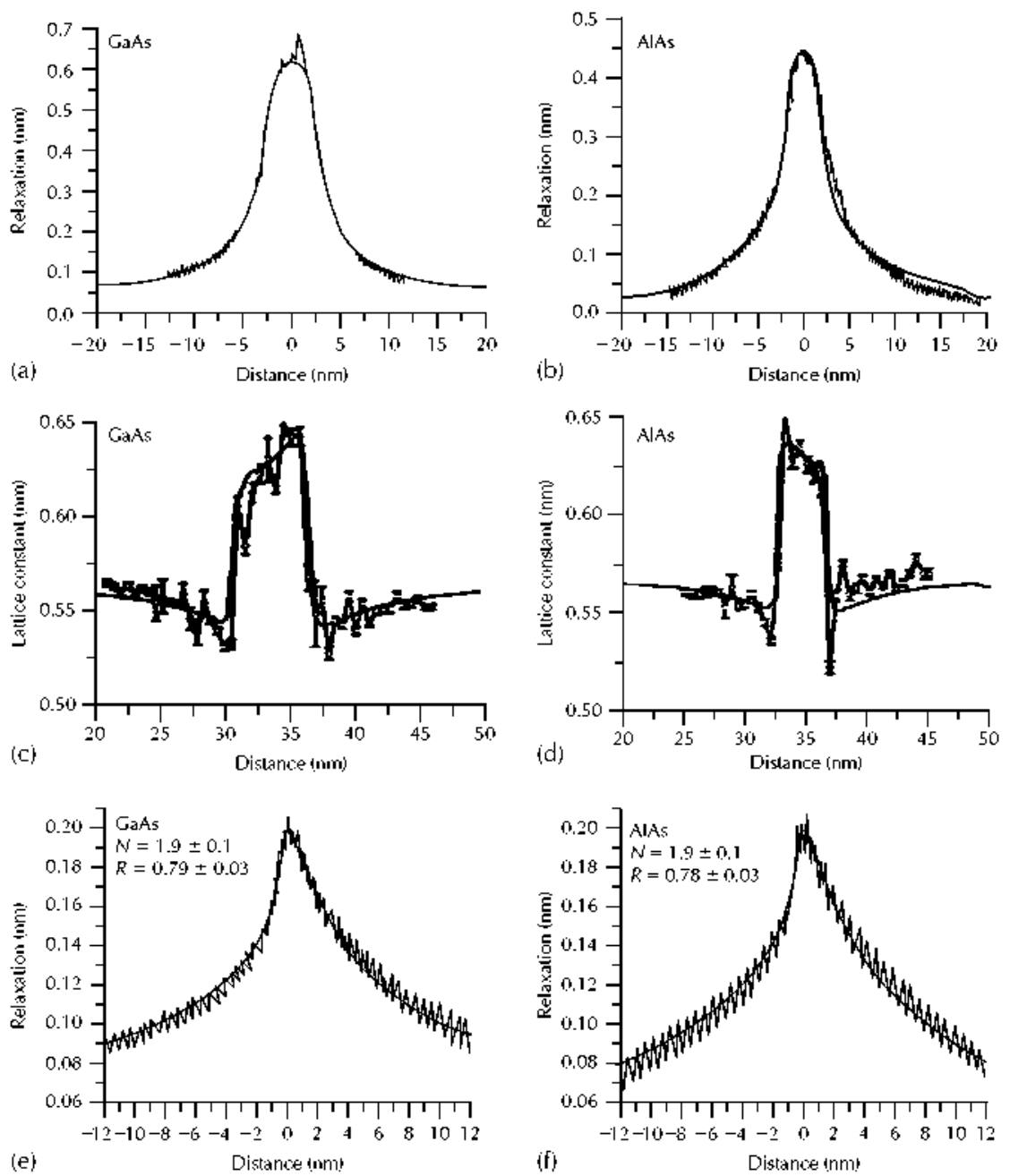

Figure 5.15 Calculated and measured relaxation profiles through the centre of the $Q D$ in the growth direction for an IhAs/GaAs $Q D$ (a) and an InAs/ALAs $Q D$ (b). (c) and (d) are the corresponding calculated and measured lat fice constant profiles. ( () ) and $(j)$ show the measured and calcuhted relaxation profiles of the WLs in GaAs and AlAs.

InAs/GaAs ODs mostly resembles the trumpet shape proposed by [26] which we describe with a linear gradient in both the growth direction and lateral direction. Along the centre of the QD, the indium concentration increases with $80 \%$ at the base to $100 \%$ at the top of the QD. The gradient in the lateral direction is dependent on position along the growth direction. At the base of the QD, it varies linearly from 80 to $40 \%$ from the core to the perimeter, while at the top of the QD it remains constant. For the InAs/AlAs QD best fit results were obtained by an indium gradient of 85 to $70 \%$ decreasing from base to top of the QD. In this case there is no indication for a lateral gradient in the indium composition.

We attribute the observed differences in indium composition and size of the QDs in GaAs and ALAs to the combined effects of (a) the reduced diffusion of In on AlAs compared to GaAs, (b) the reduced intermixing for the InAs WL on AlAs, and (c) the capping process. It is known that the indium accumulation in $\mathrm{ODs}$ is determined by strain minimization during growth. However, the preferential indium aggregation at the In-rich region of the dot is limited by the lateral diffusion of indium in the case of growth on the ALAs substrate, which results in a reduced QD size and increased QD density.

Furthermore, top-view STM experiments have shown that the surlace reconstruction of the InAs wetting layer on AlAs indicates less intermixing for the WL [52]. which together with the reduced 
mobility of In on AlAs, explains the homogeneous indium distribution in the base of the InAs/AlAs QDs. It has been proposed that the growth of dots on AlAs is initiated by 2D islands which develop into small 3D islands for higher InAs depositions [52]. This is in contrast with the growth of dots on GaAs where dot formation is initiated by small indium-rich nucleation centres which develop into trumpet-shaped indium distributions by the preferential diffusion of In to the apex of the dot [26]. These growth mechanisms are supported by our observation of the indium distribution inside the dots.

The observed decrease of the indium concentration towards the top of the InAs/AlAs QD might be caused by the residual incorporation of AlAs in the top of the dot during the capping process. In order to reduce the total strain field in the QD some capping material is incorporated in the top of the dot. In the case of GaAs capping, the diluted part of the dot is very mobile and therefore disappears very quickly during capping, causing levelling of the ODs. The InAs diluted by $\mathrm{Al}$, however, is much less mobile due to the stronger $\mathrm{Al}-\mathrm{In}$ bond strength and therefore more diluted material at the top remains in place.

In Fig. $5.15 \mathrm{e}$ and $\mathrm{f}$ we show measured and calculated relaxation profiles of segregated WLs in GaAs and AlAs that were grown at a higher temperature of $530^{\circ} \mathrm{C}$. These WLs did not show dot formation. The indium segregation profile of the WLs can be simply described by the phenomenological model of Muraki et al. described in the previous section $[27,31]$. Based on this model, we calculated the outward relaxation of the WLs using the analytical expressions by Davies et al. [24]. The optimal fit to the experimental relaxation profiles was obtained with $N=(1.9 \pm 0.1) \mathrm{ML}$, $R=0.79 \pm 0.03$ and $N=(1.9 \pm 0.1) M L, R=0.78 \pm 0.03$ for the InAs WLs in GaAs and AlAs, respectively, where $N$ is the total amount of deposited In and $R$ the segregation coefficient. The obtained values for $N$ are in agreement with the nominal deposited amount of indium. We do not find a significant difference in the segregation coefficients of indium in AlAs and GaAs as was concluded by Schowalter et al. [27]. The small apparent difference between the relaxation profiles of the WLs can be attributed to the difference in lattice constants of GaAs and AlAs. We conclude that InAs segregation does not play a role in the difference in the formation of QDs in AlAs and GaAs.

In summary. InAs QDs grown on GaAs are larger than those grown on AlAs, and they show both a lateral and a normal gradient in the In composition, while InAs/AlAs QDs show only a vertical gradient and with an opposite sign (In concentration decreasing from bottom to top). The WLs on GaAs and AlAs do not show significant differences, so we suggest that the segregation of the WL is mainly strain driven, whereas the formation of the QDs is also determined by growth kinetics. In particular, the observed differences between QDS are due to the reduced mobility of In in AlAs due to the higher Al-In bond strength, which modifies both the OD formation and capping processes.

\subsection{Capping process of InAs quantum dots}

In previous sections we have studied relevant aspects of the WL and QD formation process. Nevertheless, once created, the QDs are subsequently capped, a step which is required for any device application. Although a lot of effort has been dedicated to understand the QD growth mechanism, there are relatively few studies focused on the eflect of the capping process [12-20]. Some of these studies have already shown significant differences in size, shape and composition between uncapped and capped QDs. For example, an important collapse of the QD height has been reported for In As/GaAs ODs capped with GaAs [14, 15, 17-19], revealing the big influence of the capping process on the structural properties of the QDs.

In this section we use X-STM to analyse the capping process of InAs QDs. The effect of the capping temperature and growth interruptions is studied, as well as the impact of using different materials in the capping layer. The possibility of controlling the $\mathrm{QD}$ height with a double capping method is also discussed.

\subsubsection{Capping temperature and growth interruptions}

Levelling of InAs/GaAs QDs atter deposition of thin GaAs cap layers has been clearly revealed by top-view STM $[18,58]$ and atomic force microscopy (AFM) [54]. The levelling process has been 
attributed to the additional strain build-up between the cap layer and the partially relaxed InAs QDs $[18,54]$ to destabilize the QDs. These experiments, however, lack information about the shape and, in particular, the residual height of the QDs which is the most important parameter determining the electronic properties. In this section, we study the GaAs-capping process of InAs QDs on GaAs(100) by X-STM. The role of the capping temperature and growth interruptions is discussed. Detailed and accurate results of the QD levelling are presented, which are essential for understanding the capping process and the control of the structural and electronic properties of InAs QDs.

The samples were grown by solid source MBE on Si-doped $n$-type GaAs(100) substrates. After oxide desorption at $580^{\circ} \mathrm{C}$, a $150 \mathrm{~nm}$ thick GaAs buffer layer was grown. Then the substrate temperature was lowered to $500^{\circ} \mathrm{C}$ for deposition of $2.1 \mathrm{ML}$ InAs. Formation of InAs QDs was verified by the sharp transition from streaky to spotty of the reflection high-energy electron diffraction pattern. Thereafter, for samples A-D different capping procedures were applied: (A) deposition of $10 \mathrm{~nm} \mathrm{GaAs}$ at $500^{\circ} \mathrm{C}$ followed by $150 \mathrm{~nm} \mathrm{GaAs}$ growth at $580^{\circ} \mathrm{C}$; (B) cooling down the sample to $300^{\circ} \mathrm{C}$ before capping the InAs QDs by $10 \mathrm{~nm}$ GaAs at the same temperature and growth of $150 \mathrm{~nm}$ GaAs at $580^{\circ} \mathrm{C}$; (C) capping the InAs QDs by $3 \mathrm{ML} \mathrm{GaAs}$ at $500^{\circ} \mathrm{C}$, then cooling down the sample to $300^{\circ} \mathrm{C}$ for deposition of $150 \mathrm{~nm}$ GaAs; and (D) capping the InAs QDs by $3 \mathrm{ML}$ GaAs at $500^{\circ} \mathrm{C}$ followed by a growth interruption (GI) of time $t$, deposition of $10 \mathrm{~nm}$ GaAs at the same temperature, and growth of 50 or $150 \mathrm{~nm} \mathrm{GaAs}$ at $580^{\circ} \mathrm{C}$. In sample D five such InAs QD layers were inserted with GI times $t$ of $0,20,40,60$, and 90 seconds, separated by $60 \mathrm{~nm}$ GaAs. An extra $30 \mathrm{~nm} \mathrm{GaAs} \mathrm{spacer} \mathrm{was} \mathrm{grown} \mathrm{as} \mathrm{a} \mathrm{marker} \mathrm{between} \mathrm{the} \mathrm{QD} \mathrm{layers} \mathrm{with} \mathrm{GI} \mathrm{times} t$ of 40 and 60 seconds. The time for cooling down samples $\mathrm{B}$ and $\mathrm{C}$ from 500 to $300^{\circ} \mathrm{C}$ was 4 minutes. The growth rates were 0.58 and $0.06 \mathrm{ML} / \mathrm{s}$ for GaAs and InAs, respectively, and the $A s_{4}$ beam equivalent pressure was $1 \times 10^{-5}$ torr.

Figure 5.16a shows the filled state's topography X-STM image of the InAs QDs in sample A which are capped in the conventional way by $10 \mathrm{~nm} \mathrm{GaAs}$ at $500^{\circ} \mathrm{C}$ and $150 \mathrm{~nm} \mathrm{GaAs}$ at $580^{\circ} \mathrm{C}$. The marked part of the image is shown in Fig. $5.16 \mathrm{~b}$ after treatment with a local mean equalization filter [55] to enhance atomic details by removing the large scale background contrast. The bright horizontal lines are the top zig-zag rows of the (110) surface, which are separated by one bilayer (BL), i.e. two MLs. The topographical contrast in Fig. 5.16a is due to the outward relaxation of the cleaved surface of the compressively strained InAs QDs, revealing their cross-sectional shape. The bright spots in Fig. 5.16b correspond to In atoms in the top layer of the cleaved surface. The height of the InAs QDs in sample A is measured as 8BLs by counting the number of atomic rows.

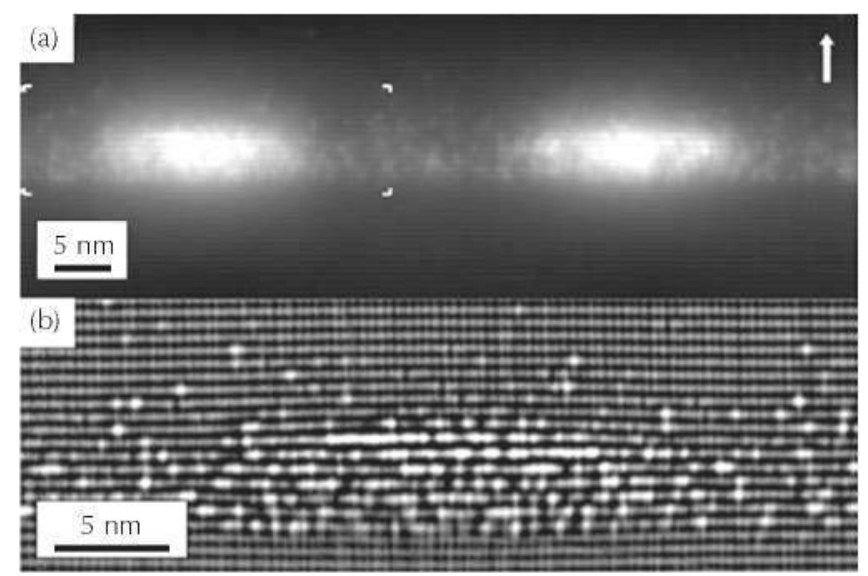

Figure 5.16 (a) Filled states topography X-STM image of the InAs QDs in sample A with conventional capping by $10 \mathrm{~nm} \mathrm{GaAs} \mathrm{at} 500^{\circ} \mathrm{C}$ and $150 \mathrm{~nm} \mathrm{GaAs}$ at $580^{\circ} \mathrm{C}$. $\mathrm{V}_{\text {sample }}=-3.0 \mathrm{~V}$. A part of the image (a) marked by four corners is treated by a local mean equalization filter and shown in $(b)$. In (a) the arrow indicates the growth direction. The black-to-white height contrast in (a) is 0 to $0.5 \mathrm{~nm}$. 
It is well established that InAs QDs buried in the conventional way of sample A exhibit a reduced height compared to unburied ones due to QD levelling $[18,53,54]$. In order to determine the QD height reduction, the shape change during overgrowth of the InAs QDs in sample B is strongly suppressed by capping them at $300^{\circ} \mathrm{C}$. The filled state's topography X-STM image of an InAs QD in sample B is shown in Fig. 5.17a with the filtered image in Fig. 5.17b. The InAs QD exhibits very sharp and well-defined interfaces confirming the suppressed QD levelling, atom diffusion, and segregation [29] and, thus, the preservation of the QD shape [56, 57]. The height of the InAs QD is 12 BLs which is 4 BLs larger than that of the QDs in sample A. This indicates that during conventional capping at $500^{\circ} \mathrm{C}$ the QD height is reduced by about one third of the original one.

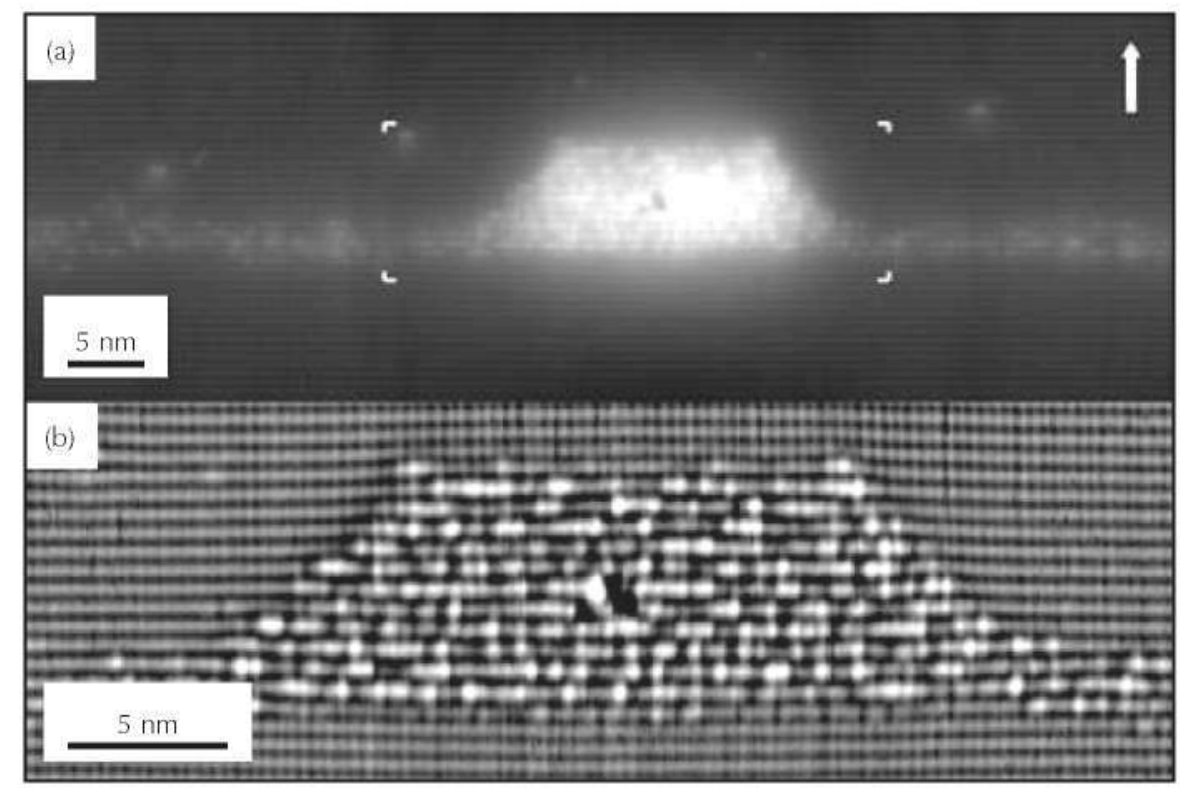

Figure 5.17 (a) Filled states topography X-STM image of the InAs QD in sample B with the GaAs cap grown at $300^{\circ} \mathrm{C} . \mathrm{V}_{\text {sample }}=-3.0 \mathrm{~V}$. A part of the image (a) marked by four corners is treated by a local mean equalization filter and shown in (b). In (a) the arrow indicates the growth direction. The black-to-white height contrast in (a) is 0 to $0.5 \mathrm{~nm}$.

When InAs QDs are capped only by a very thin GaAs layer, strong QD levelling or QD collapse occurs. Figure 5.18 shows the filled state's topography and filtered images of such InAs QDs in sample $\mathrm{C}$. The QDs are capped at $500^{\circ} \mathrm{C}$ by $3 \mathrm{ML} \mathrm{GaAs}$ and subsequently overgrown at $300^{\circ} \mathrm{C}$ to further maintain the shape. During the thin GaAs capping and cooling down, the levelling of the InAs QDs leads to a rather homogeneous (In,Ga)As layer in between the QDs due to In detachment from the QD tops, Ga/In intermixing, and In segregation with a thickness of about $4 \mathrm{BL}$, which is much thicker than the original InAs wetting layer. Intermixing with the GaAs substrate [58] expected during the growth of InAs at $500^{\circ} \mathrm{C}$ additionally contributes approximately $3 \mathrm{ML}$ of GaAs to the (In,Ga)As layer, which is derived by subtracting the thicknesses of deposited InAs (2.1 ML) and GaAs (3ML) from the total (In,Ga)As thickness of 4 BL. After levelling of the QDs, unincorporated In floating on the surface is pinned there by the low-temperature GaAs capping and forms an In-rich layer marked by the arrow in Fig. $5.17 \mathrm{~b}$. The sharp interface between this layer and the lowtemperature GaAs cap confirms that In segregation and diffusion in growth direction are strongly suppressed for $\mathrm{GaAs}$ overgrowth at $300^{\circ} \mathrm{C}$. Most interestingly, the InAs QDs are completely levelled to the thickness of the (In, Ga)As layer in between them, which is much smaller than the height of the QDs observed in sample A. This suggests that the conventional, continuous GaAs capping at $500^{\circ} \mathrm{C}$ in sample A not only drives QD levelling during the initial stage, like the thin GaAs capping in sample $\mathrm{C}$, but also quenches the levelling process when the QDs become buried. 


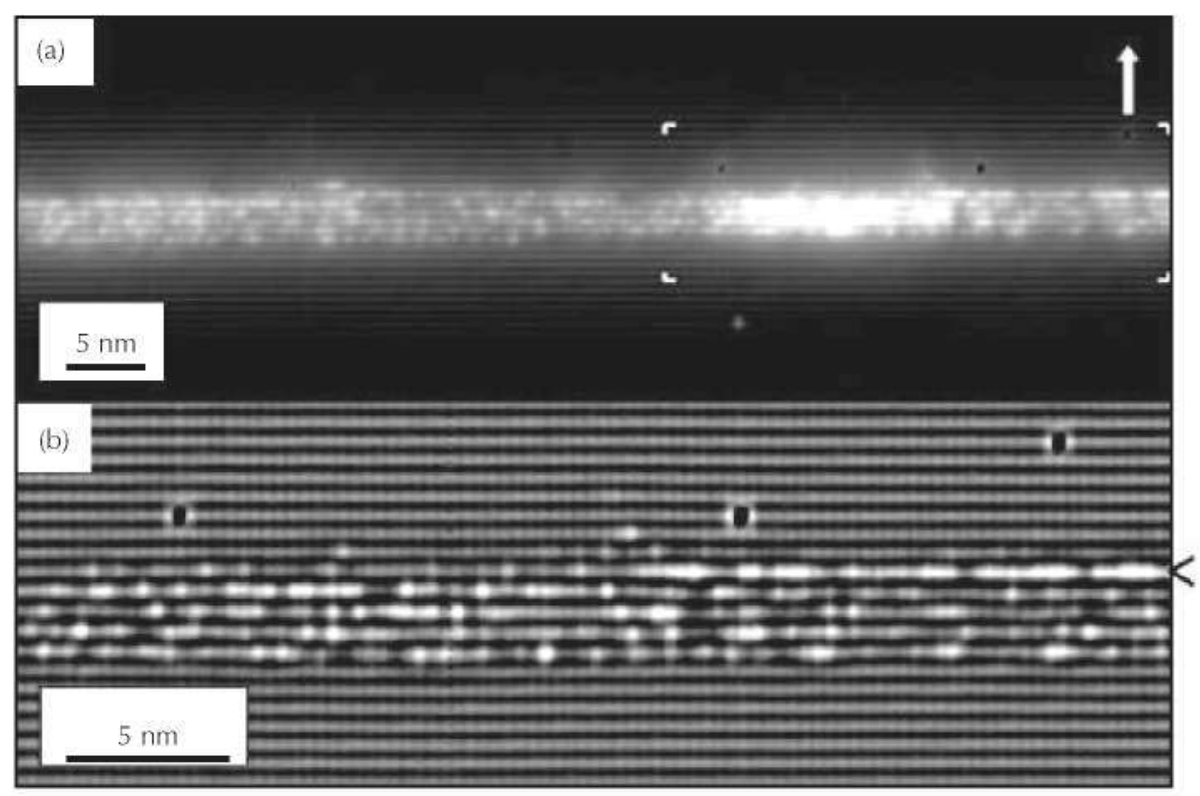

Figure 5.18 (a) Filled states topography X-STM image of the InAs QD in sample C capped by 3ML GaAs at $500^{\circ} \mathrm{C}$, followed by $150 \mathrm{~nm}$ GaAs grown at $300^{\circ} \mathrm{C}$. $V_{\text {sample }}=-3.0 \mathrm{~V}$. A part of the image (a) marked by four corners is treated by a local mean equalization filter and shown in (b). In (a) the arrow indicates the growth direction. The black-to-white height contrast in $(a)$ is 0 to $0.4 \mathrm{~nm}$. The arrow in (b) points to the In-rich layer:

To assess the time scale of QD levelling which is, thus, crucial for the final size and shape of the buried QDs, varying GI times are inserted in sample D after deposition of $3 \mathrm{ML}$ GaAs at $500^{\circ} \mathrm{C}$ on the InAs QDs prior to GaAs overgrowth. Figure 5.19 shows the filled state's topography image of the InAs QDs in sample D with GI times of 0,20,40, 60, and 90 seconds in subsequent layers. Clearly, the height of the InAs QDs capped without GI (first layer) is significantly larger than that of the QDs with insertion of 20 seconds GI. No significant further decrease in QD height is observed when the GI time is increased to 90 seconds. Hence, QD levelling during thin GaAs capping and GI takes place on a time scale of less than 20 seconds. For the present GaAs growth rate, this is comparable to the time required for growing several nanometres of GaAs to fully bury the QDs by continuous overgrowth. This indicates that both the driving and quenching of the QD levelling in conventional capping take place on a similar timescale. The size and shape of the buried QDs are therefore determined by a delicate interplay between driving and quenching of the QD levelling which is controlled by the GaAs growth rate and growth temperature.

A model based on the above experimental results is proposed for the growth of InAs QDs embedded in GaAs. The growth of InAs commences in the two-dimensional (2D) layer-by-layer mode until the InAs thickness reaches the critical value of 1.7 ML for InAs QD nucleation to reduce the accumulated strain. The InAs QDs are formed by In atoms transported massively from the 2D InAs layer, leaving a thin wetting layer on the surface. The whole system is stable at the minimum of the total energy composed of the surface energy, the strain energy, and the interface energy. Subsequent capping of the InAs QDs by GaAs, on the other hand, introduces extra strain energy between the GaAs cap and the InAs QD layer, resulting in an unstable system and the consequent QD levelling process. In atoms are redistributed from the InAs QD tops to the areas in between them during the QD levelling. They contribute to a several nanometres thick (In,Ga)As layer with an exponential In composition decay due to In segregation and $\mathrm{Ga} / \mathrm{In}$ intermixing during overgrowth [28], reducing the lattice mismatch and, hence, the total energy of the system. Thus, the thickness and the In composition profile of the (In,Ga)As layer in between the InAs QDs strongly depends on the QD levelling and In segregation. It is important to note that the QD levelling is very sensitive to the substrate temperature and is strongly suppressed at low growth temperatures, where it becomes more and more difficult to thermally break the In-As bonds. In addition to 


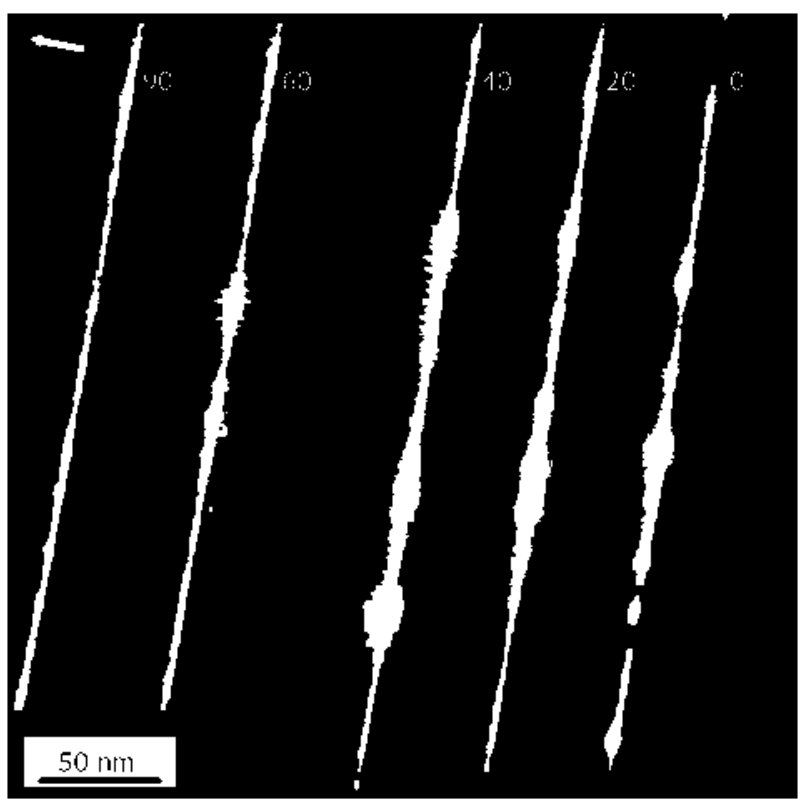

Figure 5.19 Filled states topography X-STM imnge of the five InAs QD layers in sanple D. The InAs ODs are capped by $3 \mathrm{ML}$ GaAs at $500^{\circ} \mathrm{C}$, followed by a $\mathrm{GI}$ and a $10 \mathrm{~nm}$ GaAs cap grown at $500^{\circ} \mathrm{C}$ plus a GaAs separation lajer at $580^{\circ} \mathrm{C} . \mathrm{V}_{\text {sample }}=-3.0 \mathrm{~V}$. The increasing GI times of 0.20 .40 .60 , and 90 seconds are noted in the image. The arrow indicates the growth direction. The black-to-white height contmst is 0 to $0.5 \mathrm{~mm}$.

inducing QD levelling, the GaAs cap buries the InAs QDs, thereby quenching the levelling process during continued overgrowth. Therefore, the size and shape of the embedded InAs QDs are determined by a delicate interplay between driving and quenching of the $\mathrm{QD}$ levelling during capping. which depends strongly on the growth rate and growth temperature of the GaAs cap.

In summary, we have investigated the GaAs-capping process of InAs ODs grown by molecularbeam epitaxy on GaAs(100) substrates. In its initial stage, GaAs capping induces levelling of the QDs to drastically decrease their height. During continuous capping the $Q D$ levelling is quenched when the ODs become buried. Both phenomena, driving and quenching of the OD levelling, take place on a similar time scale and are very sensitive to the GaAs growth rate and growth temperature. This understanding opens up an efficient route for controlling the size and shape of buried QDs.

\subsubsection{Capping with different materials}

We have seen how the OD structure is strongly affected by capping with GaAs and how this process is very sensitive to the growth temperature and growth interruptions. Since GaAs is the most commonly used capping material for InAs/GaAs QDs, the existing structural studies of buried InAs/GaAs QDs have been mainly devoted to GaAs-capped QDs. Nevertheless, diflerent materials such as InGaAs and GaAsSb are nowadays used to cap InAs/GaAs QDs in an eflort to extend its emission wavelength to the technologically interesting $1.3-155 \mu \mathrm{m}$ region [59-64]. For InAs/ InP QDs, capping materials other than InP, like InGaAsP, have also successtully been used for laser applications [65-67].

The use of different capping materials strongly aflects the emission wavelength and therefore should strongly affect the $\mathrm{OD}$ electronic and/or structural properties, such as size, shape, composition, strain, band offsets, etc. Indeed, critical issues occurring during capping like dot decomposition, intermixing, segregation. As/P exchange and phase separation in the capping layer depend on the capping material. To understand the impact of the capping material on the structural properties of the $\mathrm{ODs}$ is consequently of crucial importance.

In this section, we have used X-STM to analyse at the atomic scale how capping with different materials influences the structural properties of InAs QDs in GaAs and InP. The role of the 
different effects occurring during capping (intermixing, segregation, As/P exchange, compositional modulation in the capping layer, etc.) is determined. First, we study the capping with strained layers to reveal the role of strain. For that we used InAs QDs grown on (100) GaAs capped with InGaAs and GaAsSb strain reducing layers. Then we study the capping with lattice matched layers (with respect to the substrate), where the chemical effects could dominate the process. For that we study InAs dots grown on (311)B InP that were capped by either InP, InGaAs or InGaAsP (the last two materials being nominally lattice matched to InP). While strong morphological differences are found when strained layers are used, the difference between various lattice-matched capping materials is more subtle, although also relevant for device applications.

\subsubsection{Capping with strained layers}

When InAs/GaAs QDs are capped with a material which has a larger lattice constant than GaAs (and consequently closer to the one of InAs), the strain induced in the dot during capping could be smaller, which would red shift the emission wavelength. In addition, the modified strain difference could induce differences in dot size, shape and composition since dot decomposition during capping could be influenced by the strain. This is what we analyse in this section.

\subsection{InGaAs capping of InAs/GaAs QDs}

Capping with InGaAs has become popular in the last few years because it allows the emission wavelength of InAs/GaAs QDs [59-63] to be increased considerably. It is therefore very interesting to study how the structural properties of InAs QDs are affected by an InGaAs strain reducing layer.

The sample used in this study was grown by solid source MBE on an $n^{+}$Si-doped (100) GaAs substrate. 2.7 MLs of InAs were deposited at $500^{\circ} \mathrm{C}$ and $0.1 \mathrm{ML} / \mathrm{s}$ on an intrinsic GaAs buffer layer. The QD layer was capped with a nominally $6 \mathrm{~nm}$ thick $\mathrm{In}_{0.15} \mathrm{Ga}_{0.85}$ As layer grown at $500^{\circ} \mathrm{C}$ at a growth rate of $0.5 \mathrm{ML} / \mathrm{s}$.

Figure 5.20 shows a high voltage filled states image of an InAs/GaAs QD capped with InGaAs. The InGaAs layer is not well appreciated in this image because the contrast is dominated by the cleavage induced defects close to the dot. The dot is $7.0 \pm 0.5 \mathrm{~nm}$ high, considerably higher than the typical GaAs-capped QDs, which are $3-5 \mathrm{~nm}$ high, as shown in previous sections $[15,18,19]$. Nevertheless, the top facet is not as well defined as in the GaAs case and the In content decreases gradually in the growth direction (that is why the error in determining the height is larger). The In composition in the capping layer was deduced by the analysis of the outward relaxation. The fit shown in Fig. 5.21 was found when an $8.5 \mathrm{~nm}$ thick layer (as measured from the X-STM images) with $17 \%$ In content is considered in the calculation. The In content is close to the nominal value $(15 \%)$, although the layer is considerably thicker than the nominal $6 \mathrm{~nm}$. The lattice

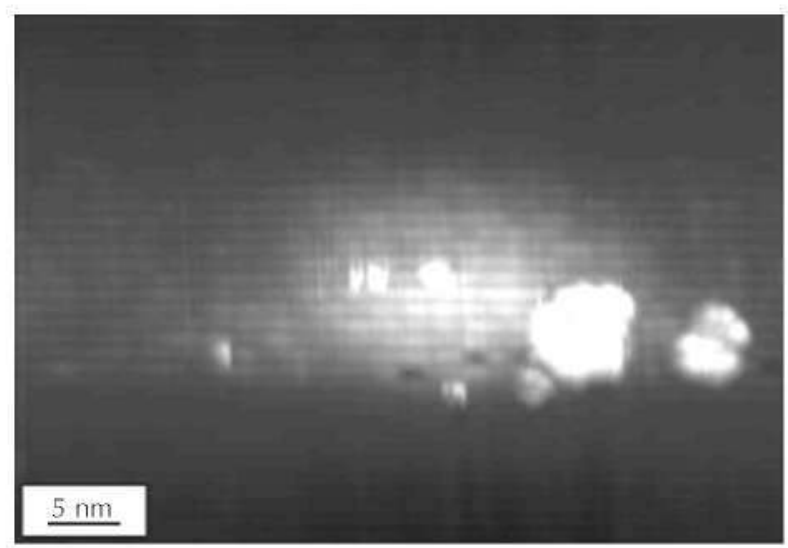

Figure 5.20 Topography image of an InAs/GaAs QD capped with InGaAs $\left(\mathrm{V}=-3 \mathrm{~V}, \mathrm{I}_{\text {tunnel }}=55 \mathrm{pA}\right)$. The big white regions are cleavage induced defects. Growth direction: [001]. lateral direction: [110]. 
mismatch between InAs and the capping layer is now 0.056 , about $17 \%$ smaller than in the case in which GaAs capping is used. We think that the reduced dot decomposition during capping is related to this smaller lattice mismatch; the result is that the dot height is increased by factor of $\sim 2$ compared to GaAs capping. This interpretation is in agreement with previously reported results in which QDs capped with InGaAs instead of GaAs are shown to retain their shape during the initial stages of capping [71, 72]. It could be argued that the increase in dot height is due to strain induced compositional modulation in the capping layer [68]. Although part of the increase in dot height could be due to that effect, we think that its contribution should be very small, since no traces of compositional modulation are observed in the capping layer. The role of the strain can be clarified by using GaAsSb as the capping material, because in this case the InAs dot height cannot increase due to phase separation in the capping layer.

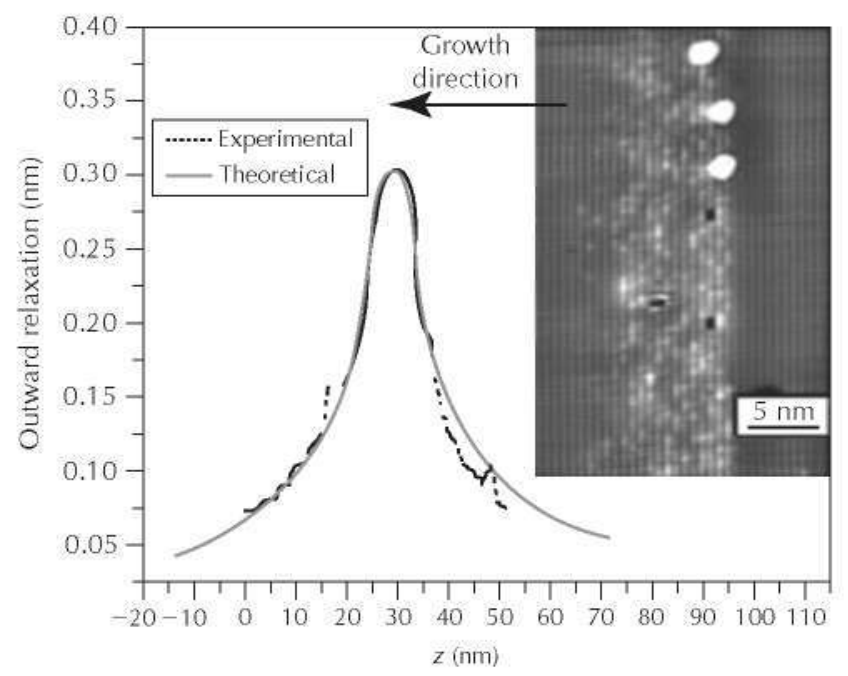

Figure 5.21 Measured (dotted line) and calculated (solid line) outward relaxation profiles of the InGaAs capping layer. A $8.5 \mathrm{~nm}$ thick layer (as measured from the images) with a $17 \%$ In content was considered in the calculation. The inset shows a high-pass filtered topography image of the InGaAs capping layer $\left(\mathrm{V}=-3 \mathrm{~V}, \mathrm{I}_{\text {tumnel }}=55 \mathrm{pA}\right)$. The big white regions are cleavage induced defects.

5.4.2.1.2 GaAsSb capping of InAs/GaAs QDs

In the last few years, GaAsSb capping layers have also been used to increase the emission wavelength of InAs/GaAs QDs [63, 69] and room temperature photoluminescence at $1.6 \mu \mathrm{m}$ has recently been reported from GaAsSb-capped $\operatorname{In}(\mathrm{Ga}) \mathrm{As} / \mathrm{GaAs}$ QDs $[64,70]$. The strong red shift observed by using GaAsSb instead of GaAs capping layers has been attributed to a type II band alignment [64, 70]. However, the structural properties of these QDs have not been studied, despite the fact that they could be significantly different from those of GaAs-capped QDs. Due to the larger lattice constant of GaAsSb compared to GaAs, the strain induced in the dot during capping could be smaller, which would further red shift the emission wavelength and could affect the dot size, shape and composition, as was the case with InGaAs capping.

The studied samples were grown by solid source MBE on $n^{+}$Si-doped (100) GaAs substrates. In the first sample (sample A), 2.7 monolayers (ML) of InAs were deposited at $500^{\circ} \mathrm{C}$ and $0.1 \mathrm{ML} / \mathrm{s}$ on an intrinsic GaAs buffer layer. The QDs were capped with a nominally $6 \mathrm{~nm}$ thick $\mathrm{GaAs}_{0.75} \mathrm{Sb}_{0.25}$ layer grown at $475^{\circ} \mathrm{C}$. In the second sample (sample B), two QD layers were grown under the same conditions $\left(2.7 \mathrm{ML}\right.$ of InAs deposited at $500^{\circ} \mathrm{C}$ and $\left.0.1 \mathrm{ML} / \mathrm{s}\right)$. The first layer was capped with $\mathrm{GaAs}$, and the second with $6 \mathrm{~nm} \mathrm{GaAs}{ }_{0.75} \mathrm{Sb}_{0.25}$. The capping temperature was $500^{\circ} \mathrm{C}$ in both cases. A layer of surface QDs was also grown for atomic force microscopy measurements. 
A number of individual QDs were analysed in sample A in order to extract information concerning their size, shape and composition. Figure 5.22 shows a high voltage filled states topography image of a QD in this sample. The group $\mathrm{V}$ elements are imaged in this measurement so that the bright spots are $\mathrm{Sb}$ atoms in the As matrix. Sb segregation into the GaAs layer is clearly observed. Contrary to what happens when capping with GaAs at similar temperatures [12], the capping layer covers all of the dot. This behaviour cannot be attributed to a larger bond strength preventing Ga migration on the growth surface, since the Ga-Sb bond is weaker than the Ga-As one $(45.9$ and $50.1 \mathrm{Kcal} / \mathrm{mol}$, respectively). The reason is likely to be the smaller strain that exists between the partially relaxed InAs QDs and the GaAsSb capping layer. This smaller strain can be accommodated by the system at the present growth temperature $\left(475^{\circ} \mathrm{C}\right)$ without inducing the migration of capping material away from the top of the dots. The dot is a full pyramid with a diagonal base length of $32 \pm 2 \mathrm{~nm}$. The height is $9.5 \pm 0.2 \mathrm{~nm}$, much larger than that of typical GaAs-capped QDs (3-5 nm high) $[15,18,19]$. This result indicates that the QDs are not dissolved during capping with GaAsSb.

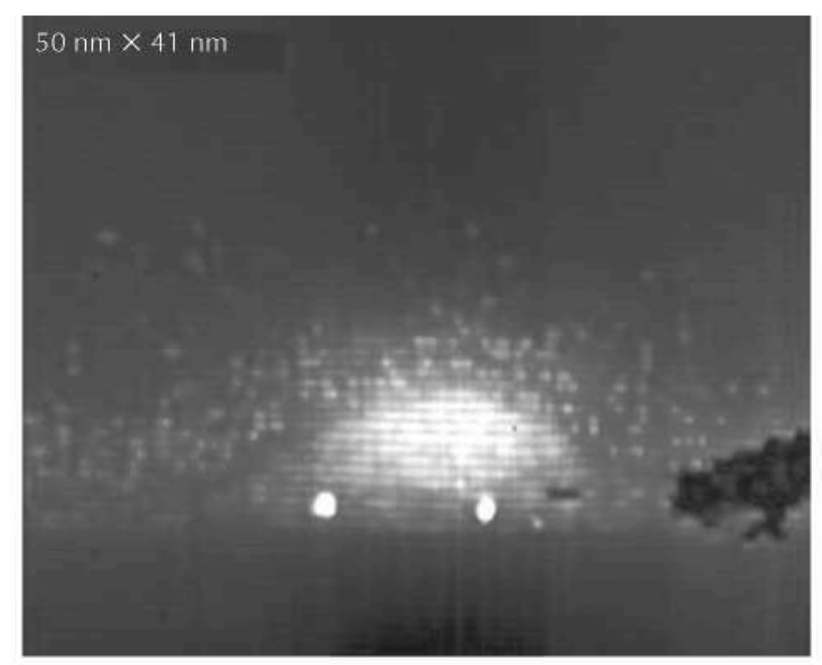

Figure 5.22 Filled states topography image of an InAs/GaAs QD capped with GaAsSb $(\mathrm{V}=-3 \mathrm{~V})$. The bright spots are Sb atoms in the As matrix. The white circles and the dark feature are cleavage induced defects.

The Sb composition in the capping layer can be obtained directly by counting the individual $\mathrm{Sb}$ atoms in the filled states images, for example Fig. 5.23a. The Sb concentration profile as a function of distance along the growth direction is plotted in Fig. 5.23b. The average Sb content is $22 \%$. This result can be checked by also analysing the outward relaxation of the cleaved surface. The relaxation of the capping layer was compared to calculations from continuum elasticity theory. The fit shown in Fig. 5.23c was obtained for a $5 \mathrm{~nm}$ thick layer (as measured from the X-STM images) with a $25 \% \mathrm{Sb}$ composition. The agreement between theory and experiment is quite good in the region of the layer itself, but deviates above the layer. This deviation is due to the effect of Sb segregation, which is not included in the model but is clearly present in the structure (see Fig. 5.23a and the asymmetry in the Sb profile obtained by atom counting). The obtained $\mathrm{Sb}$ concentration of $22-25 \%$ agrees well with the nominal growth value. The lattice mismatch between this GaAsSb composition and relaxed InAs is 0.048 , about $28 \%$ smaller than in the case in which GaAs capping is used. We propose that the fact that dot decomposition during capping is suppressed is related to the reduced strain between the dot and the capping layer, although chemical effects due to the presence of $\mathrm{Sb}$ could also be relevant. The fact that the lattice mismatch is now considerably smaller than in the InGaAs-capped sample studied before could explain the fact that the dots are now completely preserved while they are still partially dissolved when capping with $\operatorname{In}_{0.15} \mathrm{Ga}_{0.85} \mathrm{As}$, although the different chemistry in the two cases must be taken into account. 

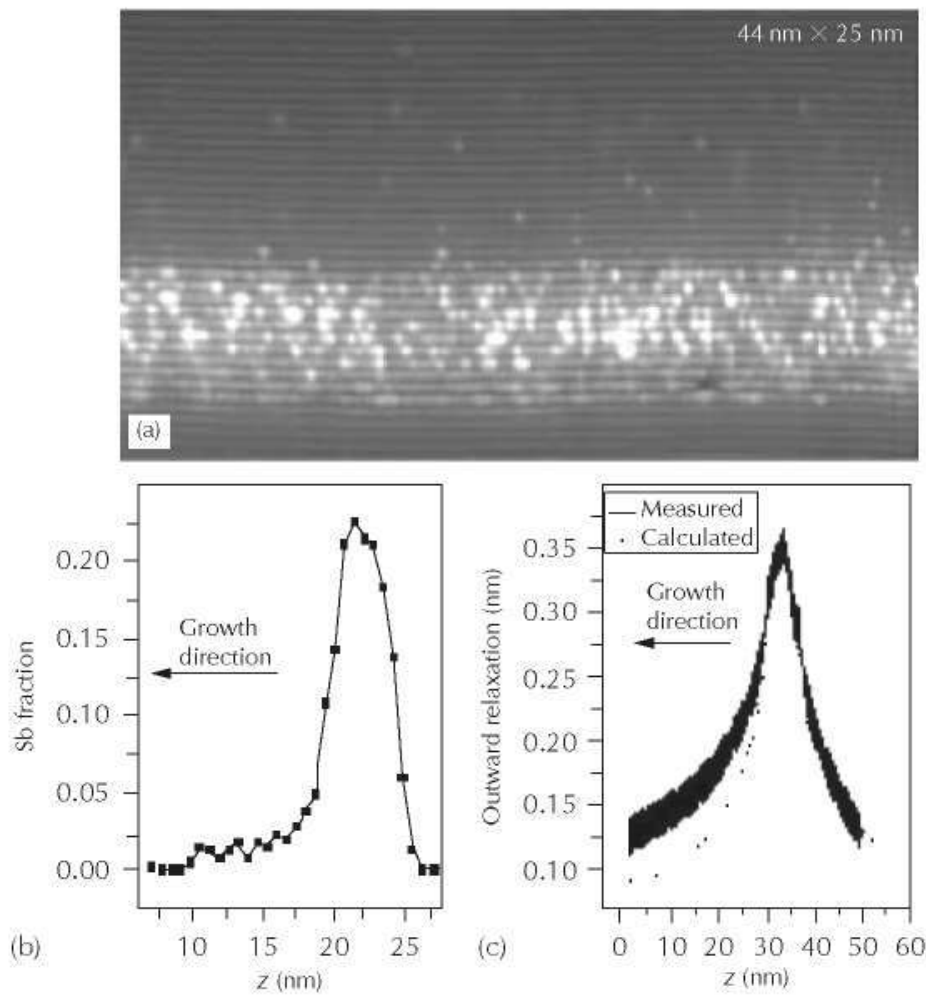

Figure 5.23 (a) Topography image of the GaAsSb capping lauer $(\mathrm{V}=-3 \mathrm{~V})$. The bright spots are Sb atoms in the As matrix. Sb segregation into the GaAs layer is clearly observed. (b) Sb concentration profile in the capping layer is obtained by counting individual Sb atoms. (c) Measured (solid line) and calculated (dotted line) outward relaxation profiles of the GaAsSb capping layer: A $5 \mathrm{~nm}$ thick layer (as measured from (a)) with a $25 \%$ Sb content was used in the calculation.

The composition of the QDs can also be estimated from an analysis of the outward relaxation. The size and shape of the dots obtained from the images are used as input parameters for the model and the composition is changed until the calculated outward relaxation profile of the dot fits the experimentally determined one. We assume that the dot with the largest observed base length is cleaved through the middle and that the dots have a square base with the edges along the $\langle 100\rangle$ directions. The largest measured baselength was $32 \mathrm{~nm}$ (see Fig. 5.22) which corresponds to a square with $23 \mathrm{~nm}$. Figure 5.24 shows the fit to the outward relaxation profile through the middle of the dot, obtained when a trumpet-like shape In composition inside the QD is included in the model. The fit deviates in the region above the dot, probably due to the fact that $\mathrm{Sb}$ segregation is not included in the calculation. The In content increases from $80 \%$ at the bottom to $100 \%$ at the top of the QD and from $40 \%$ in the corners to $80 \%$ in the centre of the base (see inset of Fig. 5.24). This is the same non-uniform In composition that we found in section 5.3 in GaAs-capped InAs/GaAs QDs [73] and similar to what has been previously proposed in [26]. It is attributed to In-Ga intermixing. The presence of In-deficient corners in the present GaAsSbcapped QDs indicates that they are not necessarily created by a redistribution of material from the top of the island to the bottom during capping, as has been recently reported [74]. In the present case, the top of the dot is not dissolved during capping but the In-poor corners are still present, hence they must originate during an earlier stage of the QD formation process.

In order to confirm the observed differences between QDs capped with GaAs and GaAsSb, sample B was studied. This sample contains QDs capped with both GaAs and GaAsSb, with both capping layers grown at the same temperature as the InAs QDs. From an analysis of the measured outward relaxation, the $\mathrm{Sb}$ concentration on the GaAsSb layer was found to be $24 \%$. However, the properties are different to those of sample A because the GaAsSb capping layer does not 


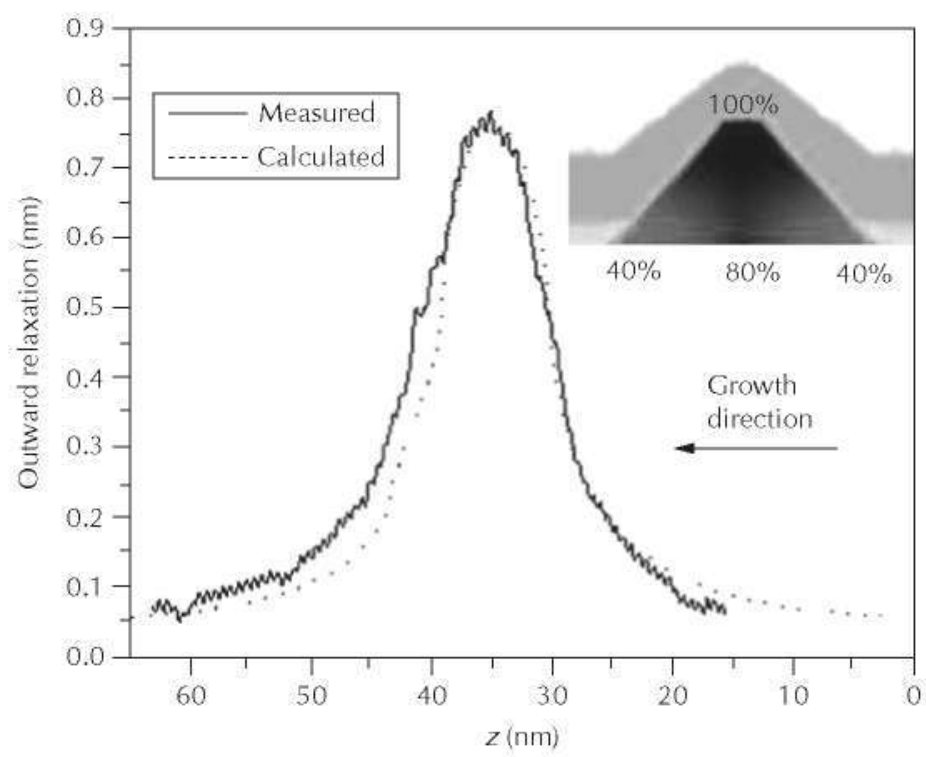

Figure 5.24 Measured (solid line) and calculated (dotted line) outward relaxation profiles through the centre of a $Q D$. The inset shows the In distribution inside the $Q D$ used in the calculation.

completely cover the dots, with their apex remaining uncovered (see Fig. 5.25a). As the only difference between samples $\mathrm{B}$ and $\mathrm{A}$ is that the capping temperature was $25^{\circ} \mathrm{C}$ higher in sample $\mathrm{B}$, it can be concluded that this increase in temperature is sufficient to allow the capping material to migrate from the relaxed apex of the dot, minimizing the strain [12]. This observation reveals that the final dot/GaAsSb capping layer configuration is very sensitive to the capping temperature. Despite this fact, differences in the QD structure for GaAs and GaAsSb capping are clearly revealed in Fig. 5.25. The GaAsSb-capped QDs (Fig. 5.25a) are again full pyramids, with a height of $8.3 \pm 0.2 \mathrm{~nm}$, while the GaAs-capped QDs (Fig. $5.25 \mathrm{~b}$ ) are truncated pyramids, with a height of only $3.8 \pm 0.2 \mathrm{~nm}$. QDs capped with GaAs are significantly dissolved during capping, while the shape of QDs capped with $\mathrm{GaAsSb}(\sim 25 \% \mathrm{Sb})$ at the same temperature is preserved. This result is confirmed by atomic force microscopy measurements on uncapped surface QDs, which are found to have a height of $8 \pm 1 \mathrm{~nm}$, in good agreement with the height of the GaAsSb-capped QDs. The base length of the strongly dissolved GaAs-capped QDs is smaller than that of the preserved GaAsSb-capped dots ( $20 \mathrm{vs} 26 \mathrm{~nm}$, respectively), indicating that the material redistribution during capping is not from the apex to the base of the dot [74], but occurs instead to the wetting layer $(\mathrm{WL})$, as was also shown in section 5.2. This is in agreement with the observation of a significantly higher In content in the WL of GaAs-capped QDs (1.8 MLs vs 0.8 MLs in GaAsSbcapped QDs), obtained by counting individual In atoms in the X-STM empty states images.

The present results indicate that strain could be playing an important role in inducing dot decomposition during capping, and that decreasing the dot/capping layer lattice mismatch could strongly reduce dot decomposition. The fact that InAs/GaAs QDs capped with GaAsSb are much bigger than those capped with GaAs should also be taken into account when explaining the observed red shift of the emission wavelength for GaAsSb-capped QDs.

In conclusion, X-STM has been used to study at the atomic scale the effect of InGaAs and $\mathrm{GaAsSb}$ capping layers on the structural properties of self-assembled InAs/GaAs QDs. QDs capped with a GaAsSb layer with 22-25\% Sb are much larger than typical GaAs-capped QDs. GaAsSbcapped QDs exhibit a full pyramidal shape of $8.3-9.5 \mathrm{~nm}$ height, while the same dots capped with GaAs are truncated pyramids with a height of only $3.8 \mathrm{~nm}$. QDs capped with $\mathrm{In}_{0.15} \mathrm{Ga}_{0.85} \mathrm{As}$ are between those two extreme cases. This finding indicates that dot decomposition during capping is suppressed by using $\mathrm{GaAs}_{0.75} \mathrm{Sb}_{0.25}$, this is likely related to the reduced dot/capping layer lattice mismatch, although chemical effects due to the presence of Sb could also be relevant. 

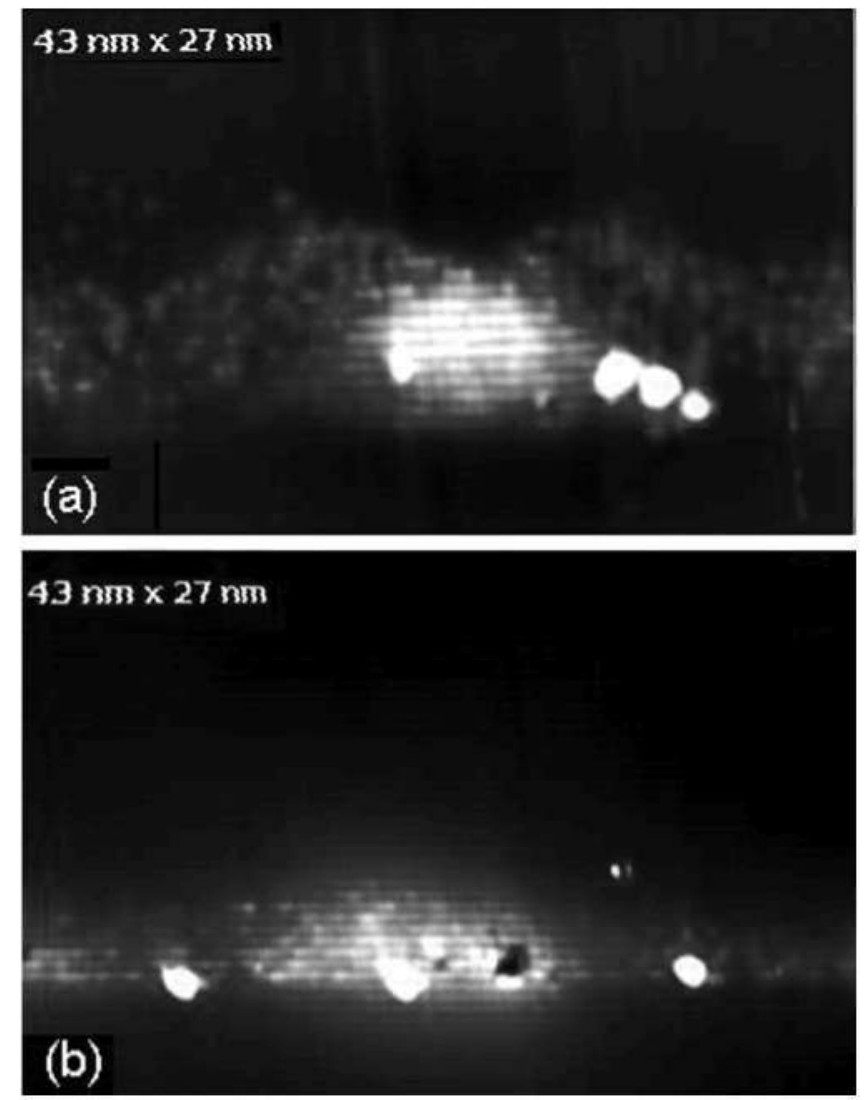

Figure 5.25 Filled states topography images of two QDs capped with (a) GaAsSb and (b) GaAs $(\mathrm{V}=-3 \mathrm{~V})$. The white circles and the dark features are cleavage induced defects.

\subsubsection{Capping with lattice-matched layers}

The results shown in the previous section seem to indicate that strain could be playing a mayor role in inducing dot decomposition during the capping process. Nevertheless, chemical effects should also be relevant, specially in the case of InAs/InP QDs, in which the As/P exchange reaction is known to be present. In order to study the chemical effects, different capping materials were used, all of them with the same lattice parameter, so the differences due to strain are eliminated.

The samples used in this study were grown by gas source MBE on an Si-doped (311)B-oriented InP substrate. This substrate orientation is very attractive for laser applications because, in comparison with InAs dot formation on conventional (100) InP substrates, a higher density of dots having a smaller size dispersion has been achieved on (311)B InP substrates $[65,66]$. Indeed, room temperature lasers with low threshold current density emitting at $1.5 \mu \mathrm{m}$ were recently demonstrated [67]. In the analysed sample the growth temperature was set to $480^{\circ} \mathrm{C}$. The QDs were formed by depositing 2.1 (100) equivalent monolayers (ML) of InAs at $0.33 \mathrm{ML} / \mathrm{s}$ on InP buffer layers. A low As flux was supplied to the surface during the InAs deposition to enhance the formation of small QDs [21]. After island formation, a 30 s growth interruption under As flux was performed before the growth of the capping layer. Three QD layers, separated by $40 \mathrm{~nm}$, were grown under the same conditions but capped with different materials: $40 \mathrm{~nm}$ of $\mathrm{InP}$ in the first layer, $20 \mathrm{~nm}$ of lattice-matched $\mathrm{In}_{0.53} \mathrm{Ga}_{0.47}$ As in the second layer (followed by $20 \mathrm{~nm}$ of $\mathrm{InP}$ ) and lattice-matched $\operatorname{In}_{0.87} \mathrm{Ga}_{0.13} \mathrm{As}_{0.285} \mathrm{P}_{0.715}$ in the third one.

A large-scale filled states X-STM image of the studied structure is shown in Fig. 5.26, in which the three QD and capping layers can be observed. The QD layers capped with InP, InGaAs and InGaAsP are labelled A, B and C, respectively. 


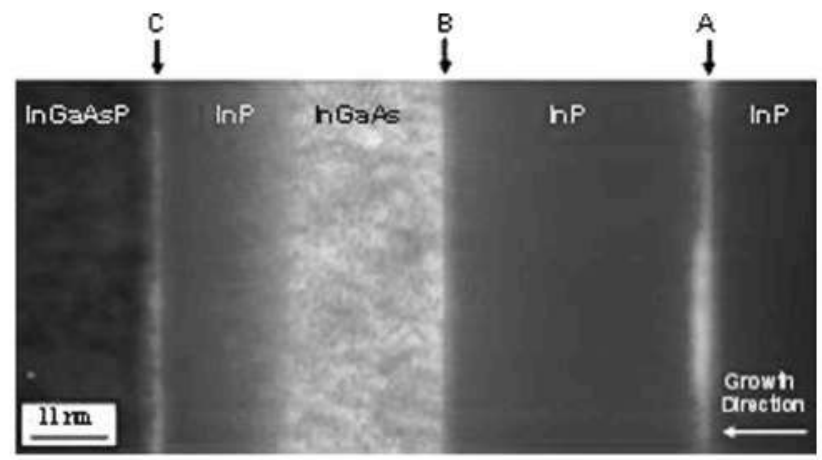

Figure 5.26 Large scale X-STM image of the structure showing the entire layer stack which comprises the InAs dot layers labelled with $A, B$ and $C\left(\mathrm{~V}_{\text {sample }}=-2.5 \mathrm{~V}, \mathrm{I}_{\text {tunnel }}=100 \mathrm{pA}\right)$. Growth direction: [113]. lateral direction: $\lceil 1 \overline{1} 01$.

A number of individual QDs were analysed within each layer in order to extract information relative to its composition, size and shape. Figure 5.27 shows the high-voltage filled states image of a single dot in layer A. Atomic details are resolved in this image, in which the group V elements, i.e. As and P, are imaged. From the homogeneity of the contrast it can be deduced that the QD composition is quite uniform and close to $100 \%$ InAs. It should be noticed that these dots are very different from the InAs/GaAs dots studied before. They resemble the shape of a quantum disc, since they have a very small aspect ratio.

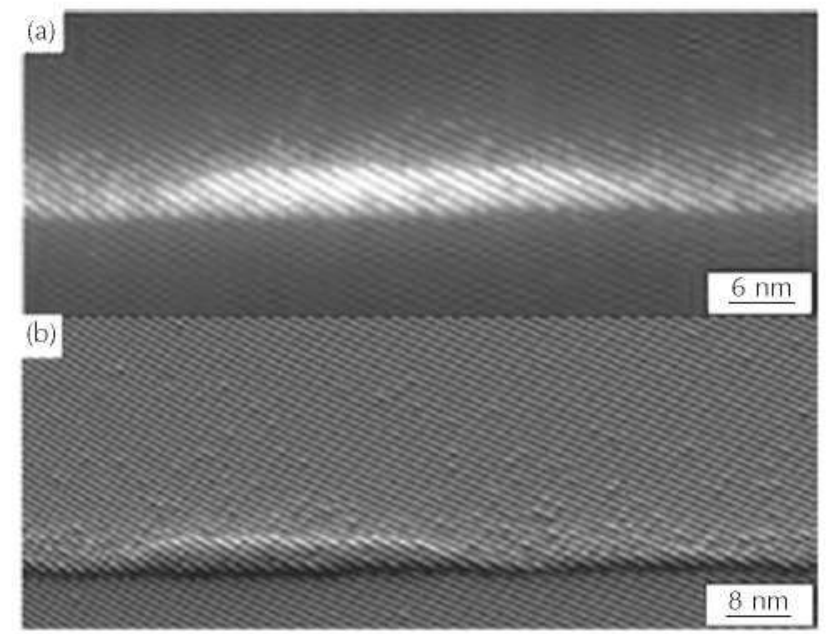

Figure 5.27 (a) X-STM topography image and (b) current image of a single InAs dot and the wetting layer (layer A) capped with $\operatorname{InP}\left(\mathrm{V}_{\text {sample }}=-3.0 \mathrm{~V}, \mathrm{I}_{\text {tunnel }}=100 \mathrm{pA}\right)$. Both of the images show the low aspect ratio of the dot having a flat top facet. In the current image As atoms can be clearly seen within the P rows of the InP capping layer. Growth direction: [113], lateral direction: [110].

All the observed dots in this layer have a similar truncated pyramidal shape, with a flat top facet. The height and base length distribution of a number of dots showed an average height of $2.9 \pm 0.2 \mathrm{~nm}$, and a maximum base length of $32 \pm 2 \mathrm{~nm}$. The difference in the outward relaxation of the dots and the WL, as indicated by the brightness in the STM image, is quite small (see Fig. 5.27a). This indicates a high As concentration in the WL. Moreover, the measured magnitude of the outward relaxation of the WL is much higher than that observed in InAs/GaAs WLs [75] (see section 5.2). This is surprising as the compressive strain in the InAs/GaAs case is more 
than twice that of the InAs/InP system and thus for equally thick wetting layers in both systems a reduction by a factor of more than two in the outward relaxation for the case of $\operatorname{InAs} / \mathrm{InP}$ should be expected. We explain this by the presence of a very thick WL which contains much more InAs material than the nominal 2.1 ML that were deposited during the dot formation.

The outward relaxation of the WL was again calculated by means of the analytical expression derived in [24], which assumes that the elastic response is linear and isotropic. The WL was modelled including the effect of the asymmetric As profile, which is likely created during the switching between phosphorus to arsenic flux or by As carryover [76]. This can be seen in Fig. 5.27b in which the bright spots in the capping layer correspond to As atoms in the InP matrix. Just as in the case of an InAs WL in GaAs, in which there is an asymmetric In profile due to segregation, we used the phenomenological model of Muraki et al. [31] to model the As profile. The total amount of deposited As is determined by fitting the calculated relaxation profile to the measured one. The result of this fitting and the corresponding As profile are shown in Fig. 5.28. We obtained a total amount of InAs of 4ML. The latter is almost twice the nominal value of deposited InAs. The origin of this extra InAs is the As/P exchange reaction at the InAs/InP interface during the dot formation process. During the growth interrupts used before and after the dot formation process, the structure is kept under a As flux promoting the exchange of $\mathrm{P}$ by As and thus increasing the amount of deposited InAs. Such exchange has also been reported to give rise to the formation of InAs QDs and InAs quantum wires on InP surfaces where only a growth interrupt was used under an As flux without the additional In deposition [77,78]. The large amount of InAs in the wetting layer is supported by the distribution of As in the WL obtained by directly counting the As atoms in the X-STM images. In Fig. 5.28 we plot the number of counted As atoms as a function of the distance in the growth direction. This method is accurate for As concentrations lower than about $25 \%$, because above those values it becomes complicated to distinguish individual As atoms in InP. The profile based on the counting is shown in Fig. 5.28 together with the profile that was used to fit the outward relaxation. The agreement is quite good in the range of validity of the counting method.

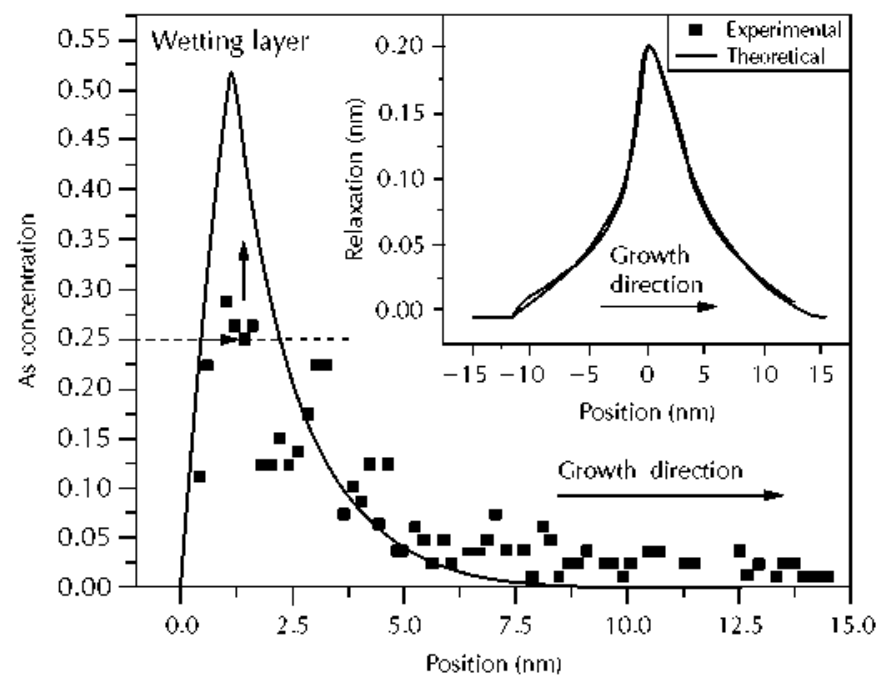

Figure 5.28 Distribution profile of As atoms from the InAs WL (huyer A). Above a concentration of $25 \%$. atom counting is not reliable and only a lower limit of the real value is obtained. The inset depicts the measured fdotted line) and the calculated (solid line) outward relaxation of the InAs wetting lajer based on the As profile that is indicated bis the solid line in the main figure.

The small height of the dots capped with InP suggests As/P exchange during the capping process. This effect should be eliminated by using InGaAs as the capping material and therefore lattice-matched $\operatorname{In}_{0.53} \mathrm{Ga}_{0.47}$ As was used in the second $\mathrm{OD}$ layer. Two dots in this layer are shown in Fig. 5.29. The height and base length distribution of a number of single dots was again 
investigated, giving an average height of $3.5 \pm 0.2 \mathrm{~nm}$ and a maximum length of $29 \pm 2 \mathrm{~nm}$. These dots are on average $0.6 \mathrm{~nm}$ higher than those capped with $\mathrm{InP}$, which corresponds to $\sim 2 \mathrm{ML}$ of InAs. This indicates that the height of the dots was reduced by $\sim 2 \mathrm{ML}$ due to dot decomposition induced by As/P exchange during $\mathrm{InP}$ capping. This value is in good agreement with the 2 ML reduction of the $\operatorname{In}(A s, P) / I n P$ quantum well width reported in [79]. The top facet of the dots capped with InGaAs is less well defined and is more curved than that of the dots capped with InP (compare Figs. 5.27 and 5.29). This is likely due to composition modulation in the capping layer, which we analyse next.

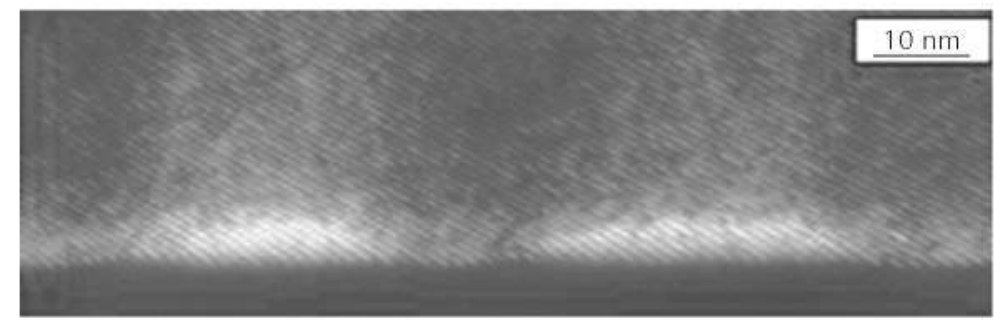

Figure 5.29 X-STM topography image of two adjacent InAs dots (in layer B) capped with InGaAs, $\left(\mathrm{V}_{\text {sample }}=-3.0 \mathrm{~V}, \mathrm{I}_{\text {tunnel }}=100 \mathrm{pA}\right)$. The image reveals the presence of a strain induced lateral phase separation in the InGaAs capping layer: Growth direction: [113], lateral direction: $[110]$.

The inhomogeneous topographic contrast in the InGaAs layer (Fig. 5.29) reveals the presence of an inhomogeneous strain distribution, which must be due to the presence of In-rich (brighter) and Ga-rich (darker) regions. This phase separation is a strain-driven process in which the In adatoms on the growth surface migrate towards the regions on top of the dots to minimize the strain, creating a columnar-like In-rich region above the dots. This process has been observed in columnar InGaAs QDs grown on GaAs [81], as well as in InAs/GaAs QDs, where capping with InGaAs has been shown to induce an increase in the dot size [68]. In our case, the phase separation directly affects the QDs by creating a rough top interface in which the In content decreases gradually.

In the third layer, a lattice-matched InGaAsP alloy was used as the capping material (Fig. 5.30). The average dot height was $3.4 \pm 0.2 \mathrm{~nm}$, and the maximum measured base length $27 \pm 2 \mathrm{~nm}$. As in the case of InGaAs, the height of the dots is higher as those capped with InP where the dot is partially dissolved due to the As/P exchange. This means that, although there is phosphorous in the capping layer, there is no dot height reduction due to As/P exchange. The shape resembles that of a truncated pyramid, with a flat top interface. More remarkable is the fact that the phase separation in the capping layer is much weaker than in the InGaAs. We think that the Ga-P bond strength $(54.9 \mathrm{kcal} / \mathrm{mol})$ which is stronger than the In-As bond strength $(48.0 \mathrm{kcal} / \mathrm{mol})$ and GaAs bond strength $(50.1 \mathrm{kcal} / \mathrm{mol})$ limits the phase segregation in InGaAsP as compared to InGaAs. The resulting weak composition modulation does not affect the dot shape or size, as is evidenced by the well-defined dot facets. Indeed, this material has been successfully used as capping layer in InAs/InP (311)B QD lasers emitting near $1.55 \mu \mathrm{m}$ with low threshold current density [67].

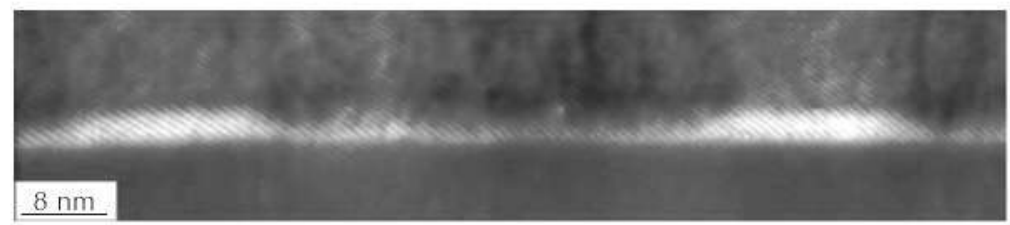

Figure $5.30 \mathrm{X}$-STM image of two InAs dots (in layer C) capped with InGaAsP alloy showing a flat base and top $\left(\mathrm{V}_{\text {sample }}=-2.5 \mathrm{~V}, \mathrm{I}_{\text {tunnel }}=100 \mathrm{pA}\right)$. Growth direction: $[113]$, lateral direction: $[1 \overline{1} 0]$.

In summary, X-STM has been used to analyse at the atomic scale the effect of different lattice-matched capping materials on the structural properties of self-assembled InAs/InP (311)B QDs. The As/P exchange on the InAs/InP interface during the growth interrupts is shown to increase the amount of InAs in the wetting layers by $\sim 2 \mathrm{ML}$. The As/P exchange takes place also 
on the dot surface when the ODs are capped with InP, reducing the dot height by about $2 \mathrm{ML}$. This phenomenon can be avoided by using InGaAs as the capping material, but in that case a strong strain-driven phase separation appears, giving rise to In-rich regions above the dots and a degradation of the dot interlace. If the quaternary alloy InGaAsP is used instead of InGaAs, the phase separation is much weaker and well-defined interlaces are obtained.

Nevertheless, the structural differences between InAs/InP QDs capped with different latticematched materials are much smaller than those found in the InAs/GaAs system when differences in strain are involved. This indicates that the dot/capping layer strain plays an important role during the capping process.

\section{4 .3 Double capping process}

We have shown in the previous section how the As/P exchange reaction can modify the InAs/InP QDs. Indeed, this effect can be intentionally used to control the height of the QDs by using a modified capping procedure which we analyse in this section. One of the main potential applications of self-assembled InAs/InP QDs is as the active region of high-performance lasers emitting at $1.55 \mu \mathrm{m}[65,67,82,83]$, the long-range optical fibre communication wavelength. For that purpose, an accurate control of the emission wavelength and of the QD size distribution is of crucial importance. One method to reduce the QD height dispersion is based on a discontinuous capping process [84], where the capping is performed in two sequences. First, a capping layer is deposited, with a thickness smaller than the $\mathrm{OD}$ height. Then, a growth interruption under phosphorous flux is performed, during which the uncovered part of the dots disappears due to As/ $\mathrm{P}$ exchange $[65,85]$. leading, in principle, to a uniform $O D$ height equal to the thickness of the first thin capping layer. Finally, the capping layer growth is completed. This modified capping procedure, named double cap (DC), was shown to allow wavelength emission control and to drastically decrease the photoluminescence (PL) linewidth [84]. Indeed, room temperature lasers based on double-capped InAs/InP QDs with low threshold current density emitting close to $1.55 \mu \mathrm{m}$ were recently demonstrated on (311)B substrates [67]. On (100) substrates, the DC process was also used for device elaboration by metal organic vapour phase epitaxy (MOVPE) [86]. However, it has been recently observed that the preferential growth of the first cap layer occurs on the edge of the QDS. This should induce an inaccurate control of the QD heights by the DC process in MOVPE [91].

The initial stage of the capping process of InAs ODs is a very complex phenomenon [12, 14, $87,88]$, sensitive to many growth parameters $[19,71]$. It has mainly been studied for InAs/ GaAs QDs, and very little is known in the case of InAs/InP QDs [80, 89-91], in which the As/P exchange reaction complicates the description. It is therefore difficult to know what happens during and after the deposition of the first capping layer in the double capping method, and a detailed structural characterization of the process is required.

We have used X-STM to study the double capping process of InAs/InP QDs grown by MBE at the atomic scale. The effect of first capping layer with different thicknesses and composition has been analysed.

The samples were grown by gas source MBE on an Si-doped (311)B-oriented InP substrate. The growth temperature was set to $480^{\circ} \mathrm{C}$. The QDs were formed by depositing 2.1 (100) equivalent MLs of InAs on both InP and $\mathrm{In}_{0.87} \mathrm{Ga}_{0.13} \mathrm{As}_{0.285} \mathrm{P}_{0.715}$ (lattice matched to InP) buffer layers. In order to make the analysis easier, a high As beam equivalent pressure was supplied to the surface during the InAs deposition to favour the formation of large QDs [66]. After island formation, a $30 \mathrm{~s}$ growth interruption under As flux was performed before the growth of the capping layer. Then, the first capping layer (CL1) was deposited and the growth was interrupted for 60 s maintaining a group V overpressure. Finally, a thick $(50$ or $100 \mathrm{~nm}$ ) second capping layer (CL2) was grown. Three different samples were studied. In sample A, three QD layers were grown with dil'erent thicknesses of CL1: 1.5, 2.5 and $3.5 \mathrm{~nm}$ (from now on called layer 1, layer 2 and layer 3 , respectively). The buffer and the capping layers consisted of $\operatorname{In}_{0.87} \mathrm{Ga}_{0.13} \mathrm{As}_{0.285} \mathrm{P}_{0.715}$. In sample $\mathrm{B}$, the buffer, CL1 and CL2 were made of InP. Finally, in sample $C_{+}$the buffer and CL2 were made of InP, while CL1 consisted of $\operatorname{In}_{0.87} \mathrm{Ga}_{0.13} \mathrm{As}_{0.285} \mathrm{P}_{0.715}$. In samples $\mathrm{B}$ and $\mathrm{C}$ four $Q D$ layers were grown with different thicknesses of CL1: 2, 3, 4 and $5 \mathrm{~nm}$ (layers $1,2,3$ and 4 , respectively). 
First, we present results on sample A, with InGaAsP buffer and capping layers. Figure 5.31 shows a large-scale filled states image of this sample. The three QD layers separated by $\sim 50 \mathrm{~nm}$ are observed. The inhomogeneous contrast in the buffer and capping layers is due to the random alloy fluctuations in the InGaAsP. Remarkably, the QDs have a very large base length. The maximum measured base length was $60 \mathrm{~nm}$, which gives aspect ratios as low as 0.04 . It is clear from Fig. 5.31 that the height of the QDs increases when the thickness of CL1 increases. Moreover, the height of the QDs in each layer fits very well with the nominal thickness of CL1. After analysing 37 dots in this sample, the measured average height in layer 1 (CL1 $=1.5 \mathrm{~nm}$ ) was $1.6 \mathrm{~nm}$, in layer 2 $(\mathrm{CL} 1=2.5 \mathrm{~nm}) 2.5 \mathrm{~nm}$, and in layer $3(\mathrm{CL} 1=3.5 \mathrm{~nm}) 3.4 \mathrm{~nm}$ (see the triangles in Fig. 5.34). This indicates that the double capping procedure is controlling the QD height with high accuracy.

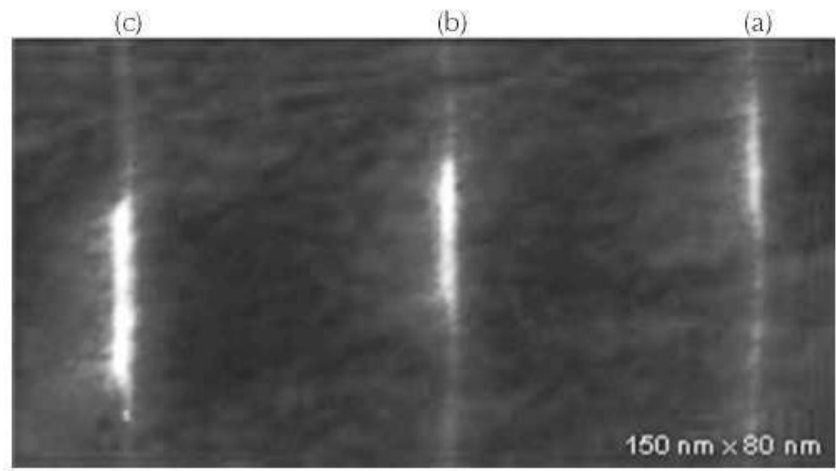

Figure 5.31 Large scale filled states image of sample A showing the three QD layers in which CL1 is 1.5, 2.5 and $3.5 \mathrm{~nm}$ thick in (a), (b) and (c), respectively. The growth direction is from right to left. $\mathrm{V}_{\text {sample }}=-2.5 \mathrm{~V}$. $\mathrm{I}_{\text {tunnel }}=50 \mathrm{pA}$.

In sample B, buffer and both capping layers consist of InP and the thickness of CL1 was increased up to $5 \mathrm{~nm}$. Figure 5.32 shows the current image of one dot in layer 2 of this sample. On such specific QDs, shallow trenches in the InP buffer close to the QDs are observed. We assume that the trenches are mainly formed just after the dot formation during the growth interrupt under As pressure. Mass transport from wetting layer to quantum dots leads to the formation of a dewetted InP surface on which As/P exchange reaction occurs. The extra InAs formed migrate to the dots and a trench is formed around the QDs. The fact that these trenches were not present in sample A (see Fig. 5.1), where the dots were grown on InGaAsP, supports this explanation (As/P exchange is reduced on an InGaAsP surface) [90]. Similar observations have been previously reported for InAs QDs grown on (001) InP, and explained in a similar way [92].

Figure 5.32 is representative of what was observed when CL1 was 2 or $3 \mathrm{~nm}$ thick. The dots are similar to the ones in sample A: they have a flat top facet and their height fits very well with the thickness of CL1 (the average height was 2.2 and $2.9 \mathrm{~nm}$ in layers 1 and 2, respectively). Nevertheless, the situation is different when CL1 is 4 and $5 \mathrm{~nm}$ thick. In this case, the measured average heights of the QDs are 3.1 and $4 \mathrm{~nm}$, respectively. Thus the QD heights are not accurately controlled by the first cap layer thickness in this range. These results confirm the PL measurement as a function of the thickness of CL1 previously published [84]. For CL1 thicker than $4 \mathrm{~nm}$, the PL energy control by the DC process is lost.

On sample C, CL1 and CL 2 consist of InGaAsP and InP, respectively. The thicknesses of CL1 were set at 2, 3, 4 and $5 \mathrm{~nm}$. Figure 5.33 shows a filled states topography image of layers 2,3 and 4 . Atomic details are resolved in this image, in which the group V elements, i.e. As and P, are imaged. As the QDs are grown on $\mathrm{InP}$, the trenches are present again, but it is clear from image $5.33 \mathrm{c}$ that it is a global effect affecting the whole WL. CL1 appears as a dark region with inhomogeneous contrast between the WL and CL2. A bright region in CL1 on top of the QDs is observed in layers 3 and 4, indicating an As- and/or In-rich region. This is likely due to strain induced composition modulation, similar to what was found in the previous section for InGaAs capping [90]. The thickness of CL1 in layers 2, 3 and 4, measured from the top of the WL, is $2.9,3.8,4.8 \mathrm{~nm}$, respectively. 


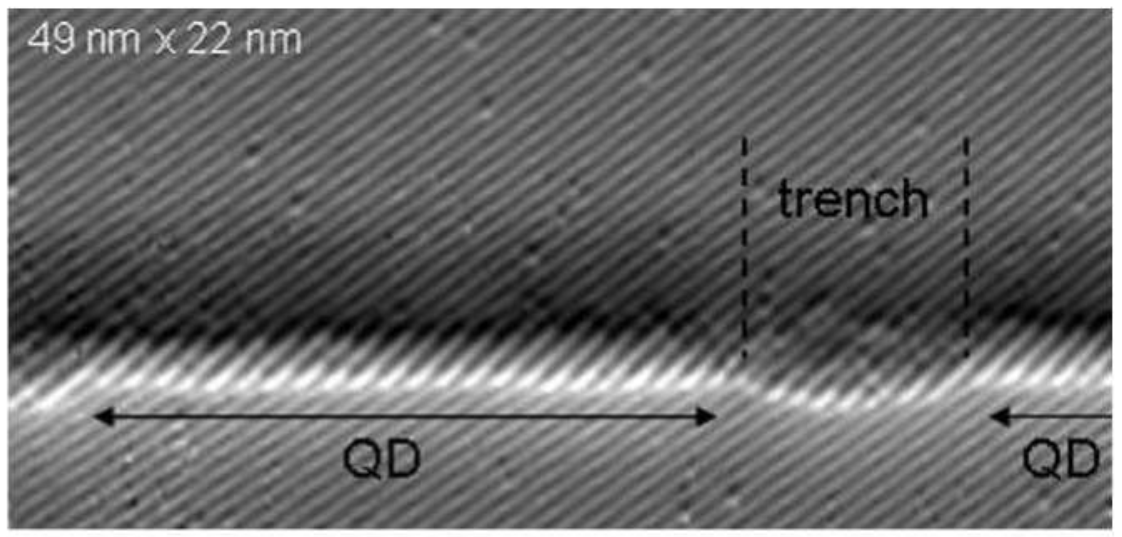

Figure 5.32 Current image of $a Q D$ in sample $B$ corresponding to layer $2(C L 1=3 \mathrm{~nm})$. Part of a second $Q D$ appears on the right and a shallow trench is clearly observed between the two $\mathrm{QDs} . \mathrm{V}_{\text {sample }}=-2.5 \mathrm{~V}, \mathrm{I}_{\text {tunnel }}=52 \mathrm{pA}$.

This is in good agreement with the nominal values. Figure 5.33 a shows how in layer 2 the height of the QD is levelled to the thickness of CL1. This is also the case in layer 1 (not shown). As in the previous sample, when the thickness of CL1 is increased to $4 \mathrm{~nm}$, the height of the QDs is slightly smaller than CL1 (see Fig. 5.33b). This difference is much bigger when CL1 is $5 \mathrm{~nm}$ thick. In this case the height of the QD is more or less half of the thickness of CL1, as shown in Fig. 5.33c. Moreover, a strong decrease of the QD density is observed in this layer. Complete island dissolution, as previously observed for large InGaAs QDs on GaAs, may be effective in this case [93]. Complementary experiments to clarify this question are still in progress.
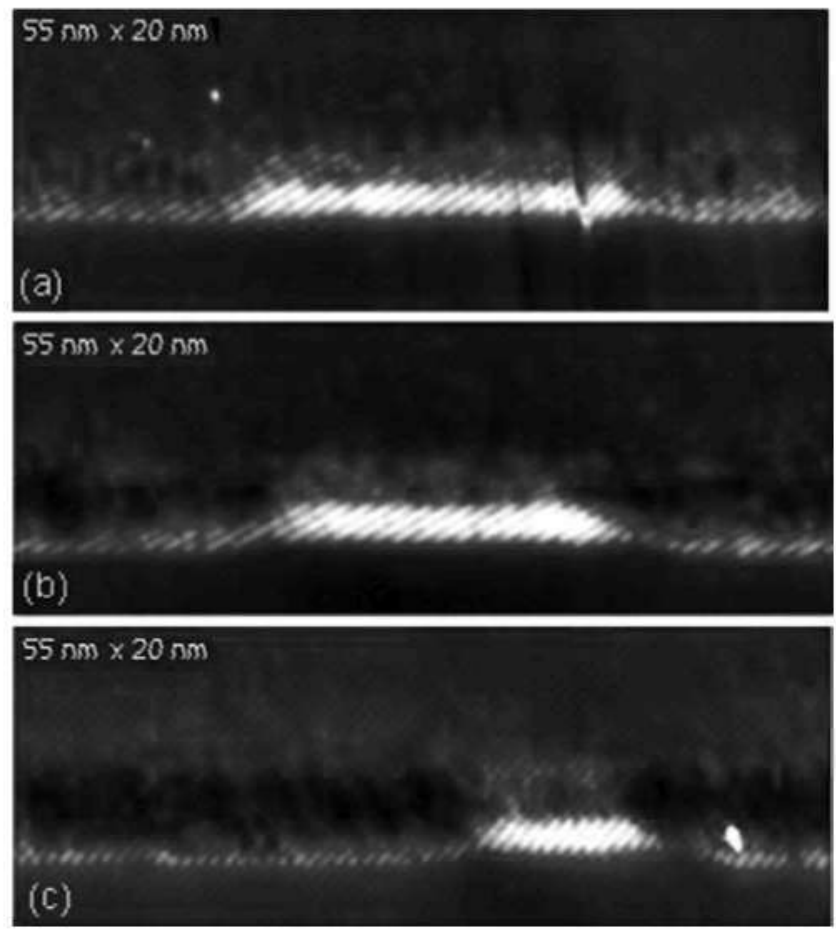

Figure 5.33 Topography image of three QDs in sample C corresponding to (a) layer 1 (CL1 $=2 \mathrm{~nm}$ ), (b) layer 3 $(\mathrm{CL1}=4 \mathrm{~nm})$ and $(c)$ layer $4(C L 1=5 \mathrm{~nm})$. CL1 appears as a dark region between the WL and CL2. The bright spots are As atoms in the P matrix. $V_{\text {sample }}=-3.0 \mathrm{~V}, \mathrm{I}_{\text {tunnel }}=53 \mathrm{pA}$. 
All the results are summarized in Fig. 5.34, in which the average height of the ODs in each layer is plotted as a function of the nominal thickness of CL1 for the three analysed samples. The solid curve represents the ideal situation where the dot height corresponds exactly to the thickness of CL1. The double capping method works well for first capping layers up to $\sim 3.5 \mathrm{~nm}$, independently of the materials used. This is very important because the desired $Q D$ height for $1.55 \mu \mathrm{m}$ applications $(\sim 2.7 \mathrm{~nm})$ lies in that range. For thicker layers, a reduction in the dot height compared to the thickness of the first capping layer is observed, indicating that the control over the QD height with the DC process is lost for capping layers thicker than $-3.5 \mathrm{~nm}$. This can be understood in terms of a transition from a double capping to a classical (one-step) capping process when the first capping layer is thick enough to completely cover the dots. Once the dots are covered, the growth interrupt has no effect on them. Since the height of similar classically capped $Q D$ s has been measured in the previous section to be around $3.5 \mathrm{~nm}$, that should be the maximum possible height obtained and the DC method should not work for CL1 thicker than that. Indeed, $3.5 \mathrm{~nm}$ is approximately the saturation value observed for thick capping layers if Fig. 5.34 (except for the special case of $5 \mathrm{~nm}$ in sample $C_{\text {. }}$ in which a strong decrease of the QD density is also observed).

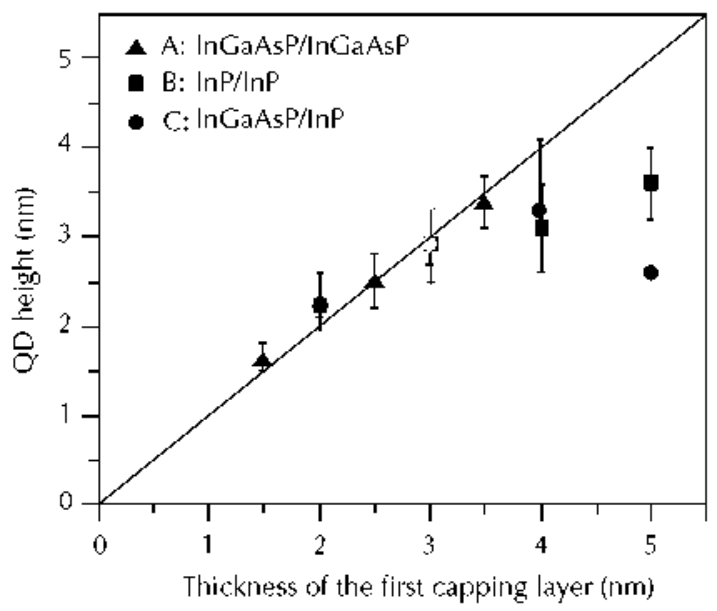

Figure 5.34 Averige $Q D$ height in each hiyer is a function of the inminal thickness of CL1 for the three analysed samples. The enor is taken as the distance to the maximum and minimum values measured. The solid curve represents the idenl siutuation where the dot height corresponds exactly to the thickness of CL1. A significunt deviation is observed for thick first capping lajers.

In summary our X-STM measurements show that the double capping method applied to InAs/ InP (311)B QDs grown by MBE allows a very accurate control of the QD height when the thickness of the first capping layer is not higher than $\sim 3.5 \mathrm{~nm}$, independently of the material used in the capping layer. For higher values a significant reduction of the $\mathrm{OD}$ height is observed. This is explained by the fact that $3.5 \mathrm{~nm}$ is the height of classically capped $Q D$ s and therefore for thicker capping layers the double capping process becomes a classical capping process since the QDs would be completely covered by the capping layer before the growth interrupt.

\subsection{Conclusions}

X-STM has been used to study at the atomic scale the formation of self-assembled QDs. This technique allows determining the size, shape and composition of the buried nanostructures and provides vital information to understand the $\mathrm{OD}$ formation process.

X-STM measurements show that the size and composition profile of InAs self-assembled quantum dots depend strongly on the substrate on which the dots are formed. In particular. InAs QDs 
grown on GaAs are larger than those grown on AlAs, and the In gradients inside the dot from bottom to top have opposite signs (increasing in InAs/GaAs ODs and decreasing in InAs/AlAs QDs) [7.3]. These diflerences are mainly due to the reduced mobility of In in AlAs due to the stronger Al-In bond strength.

The capping process is found to be a critical step in QD formation because it strongly modifies the QD structure. In its initial stage, GaAs capping induces leveling of the QDs to drastically decrease their height. During continuous capping the QD leveling is quenched when the QDs become buried. Both phenomena, driving and quenching of the QD leveling take place on a similar time scale and are very sensitive to the GaAs growth rate and growth temperature (decreasing the capping temperature can strongly reduce dot decomposition) [19]. This dot decomposition during capping can also be modified by using different capping materials [94]. It is strongly reduced when the dots are capped with $\mathrm{In}_{0.15} \mathrm{Ga}_{0.85} \mathrm{As}$ and completely suppressed when $\mathrm{GaAs}_{0.75} \mathrm{Sb}_{0.25}$ is used [95]. The result is that ODs capped with these strain reducing layers are much taller than GaAs-capped $Q D$ D. This suggests that the dot/capping layer strain plays a mayor role in inducing dot decomposition during capping. When the different capping materials are lattice matched to the substrate, the differences in the QD properties are more subtle and can be dominated by chemical effects: InAs/InP QDs capped with InP have a 2 MLs smaller height than those capped with InGaAs or InGaAsP due to As/P exchange induced decomposition [90]. The effect of the As/P exchange can be intentionally used to control the QD height by using a double capping method. We found that the double capping method allows a very accurate control of the QD height when the thickness of the first capping layer is not higher than $\sim 3.5 \mathrm{~nm}$. For higher values a significant reduction of the $\mathrm{OD}$ height is observed. These results are interpreted in terms of a transition from a double capping to a classical capping process when the first capping layer is thick enough to completely cover the dots [96].

\section{Acknowledgements}

The authors would like to specially thank the growers of the analysed samples: $Q$. Gong and R. Nötzel, COBRA-Department of Applied Physics. Eindhoven University of Technology. The Netherlands; A. Letoublon and N. Bertru, Laboratoire d'études des nanostructures semiconductrices (LENS), INSA-Rennes, France; M.J. Steer, H.Y. Liu, and M. Hopkinson, Department of Electronic and Electrical Engineering. University of Sheffield, UK; K. Pierz, PhysikalischTechnische Bundesanstalt, Germany. The authors would also like to thank John H. Davies for help with the strain calculations and Peter Maxime and Marvin Roy for help with the ABAQUS soltware. Thanks also to C. Selebi. I. Drouzas, A. Simon. and E. Gapihan for helping with the measurements and the interpretation of the data and to D.J. Mowbray for helpful discussion. This work has been supported by the European Union through the SANDiE Network of Excellence (contract No. NMP4-CT-2004-500101).

\section{References}

1. D. Bimberg. M. Grundmann, and N.N. Ledentsov, Qutuntum Dot Heterostructures (Wiley, Chichester, UK, 1999).

2. M.S. Skolnick and D.J. Mowbray. Annu. Rev. Mater: Res. 34. 181 (2004).

3. S. Fatard, K. Hinzer, S. Raymond, M. Dion, J. McCalfrey, Y. Feng, and S. Charbonneau, Science 274, $1350(1996)$.

4. F. Heinrichsdorff, M.-H. Mao, N. Kirstaedter, A. Krost, D. Bimberg, A.O. Kosogov, and P. Werner, Appl. Phys. Lett. 71, 22 (1997).

5. K. Yano, T. Ishii, T. Hashimoto. T. Kobayashi, F. Murai, and K. Seki, IEEE Trans. Electron. Devices 41. 1628 (1994).

6. S.Y. Wang, S.D. Lin, H.W. Wu, and C.P. Lee, Appl. Phys. Lett. 78, 1023 (2001).

7. A.D. Stiff, S. Krishna, P. Bhattacharya, and S.P. Kennerly. Appl. Phys. Lett. 79.421 (2001). 
8. P. Michler, A. Kiraz, C. Becher, WV. Schoenfeld, P.M. Petroff, L. Zhang. E. Hu, and A. Imamogglu, Science 290, 2282 (2000).

9. Z. Yuan, B.E. Kandynal, R.M. Stevenson, A.J. Shields, C.J. Lobo, K. Cooper, N.S. Beattie, D.A. Ritchie, and M. Pepper, Sicience 295, 102 (2002).

10. D.J. Eaglesham and M. Cerullo, Phws. Rev. Lett. 64, 1943 (1990).

11. J. Stangl, V. Holy, and G. Bauer, Rev. Mod. Physics 76. 725 (2004).

12. Q. Xie, P. Chen, and A. Madhukar, AppI. Phys. Lett. 65, 2051 (1994).

13. X.W. Lin. J. Washburn, Z. Liliental-Weber, E.R. Weber, A. Sasaki, A. Wakahara, and Y. Nabetani, Appl. Phys. Lett. 65, 1677 (1994).

14. J.M. Garcia, G. Medeiros-Ribeiro, K. Schmidt, T. Ngo, J.L. Feng, A. Lorke, J. Kotthaus, and P.M. Petrolf, Appl. Phys. Lett, 71, 2014 (1997).

15. G.D. Lian, J. Yuan, L.M. Brown, G.H. Kim, and D.A. Ritchie, Appl. Phys. Lett. 73, 49 (1998).

16. P.D. Siverns, S. Malik, G. McPherson, D. Childs, C. Roberts, R. Murray, and B.A. Joyce, Phys. Rev. B. 58, R10127 (1998).

17. R. Leon, J. Wellman, X.Z. Liao, J. Zou, and D.J.H. Cockayne, Appl. Phys. Lett. 76, 1558 (2000).

18. P.B. Joyce, T.J. Krzyzewski, P.H. Steans, G.R. Bell, J.H. Neave, and T.S. Jones, Surf. Sci. 492, 345 (2001).

19. Q. Gong, P. Offermans, R. Nötzel, P.M. Koenraad, and J.H. Wolter Appl. Phus. Lett. 85, 5697 (2004).

20. G. Costantini, A. Rastelli, C. Manzano, P. Acosta-Diaz, R. Songmuang, G. Katsaros, O.G. Schmidt, and K. Kern, Phys. Rev. Lett. 96, 226106-1 (2006).

21. G. Binning, H. Rohrer, Ch. Gerber, and E. Weibel, Appl. Phys. Lett. 40, 178 (1982).

22. R.M. Feenstra, Physica B. 273, 796 (1999).

23. D.M. Bruls, P.M. Koenraad, M. Hopkinson, J.H. Wolter, and H.W.M. Salemink, Appl. Surf. Sci. 190. $258(2002)$

24. J.H. Davies, D.M. Bruls, J.W.A.M. Vugs, and P.M. Koenraad, J. Appl. Phys. 91,4171 (2002).

25. K. Sautho, M. Wenderoth, A.J. Heinrich, M.A. Rosentreter, K.J. Engel, T.C.G. Reusch, and R.G. Ulbrich, Phys. Rev. B. 60, 4789 (1999).

26. M.A. Migliorato, A.G. Cullis, M. Fearn, and J.H. Jefferson, Phys. Rev. B. 65, 115316 (2002).

27. M. Schowalter, A. Rosenauer, D. Gerthsen, M. Arzberger, M. Bichler, and G. Abstreiter, App. Phys. Lett. 79.4426(2001).

28. A. Rosenauer, D. Gerthsen, D.V. Dyck, M. Arzberger, G. Böhm, and G. Abstreiter, Phys. Rev. B. 64, $245334(2001)$.

29. J.P. Silveira, J.M. García, and F. Briones, AppI. Surf. Sci. 188, 75 (2002).

30. J.M. Moison, C. Guille, F. Houzay, F. Barthe, and N.V. Rompay, Phys. Rev. B. 40, 6149 (1989).

31. K. Muraki, S. Fukatsu, Y. Shiraki, and R. Ito, Appl. Phys. Lett. 61, 557 (1992).

32. N. Grandjean, J. Massies, and M. Leroux, Phys. Rev. B. 53,998 (1996).

33. O. Dehaese, X. Wallart, and F. Mollot, Appl. Phys. Lett. 66, 52 (1995).

34. A.G. Cullis, D.J. Norris, T. Walther, M.A. Migliorato, and M. Hopkinson, Phys. Rev. B. 66 . $081305(\mathrm{R})(2002)$.

35. A. Lenz, R. Timm, H. Eisele, C. Hennig, S.K. Becker, R.L. Sellin, U.W. Pohl, D. Bimberg, and M. Dähne, Appl. Phys. Lett. 81, 5150 (2002).

36. K. Pierz, Z. Ma, U.F. Keyser, and R.J. Haug, J. Crisst. Growth 249, 477 (2003).

37. P. Offermans, P. Koenraad, J. Wolter, K. Pierz, M. Roy, and P. Maksym, Physica E. 26, 236 (2005).

38. Y.C. Kao, F.G. Celli, and H.Y. Liu, J. Vac. Sci. Technol. B. 11, 1023 (1993).

39. D. Granados and J.M. García, Appl. Phys. Lett. 82. 2401 (2003).

40. L.G. Wang. P. Kratzer, M. Scheffler, and O.K.K. Liu, Appl. Phys. A. 73, 161 (2001).

41. Z.R. Wasilewski, S. Fatard, and J.P. McCatfrey, J. Cryst. Grouth 201/202, 1131 (1999).

42. E. Steimetz, T. Wehnert, H. Kirmse, F. Poser, J.-T. Zettler, W. Neumann, and W. Richter, J. Cryst. Grouth 221,592 (2000).

43. A. Lenz, H. Eisele, R. Timm, S.K. Becker, R.L. Sellin, U.W. Pohl, D. Bimberg, and M. Dähne, Appl Phys. Lett. 85, 3848 (2004).

44. I. Hapke-Wurst et aI., Semicond. Sci. Technol. 14, L41-L43 (1999).

45. K. Pierz, Z. Ma, U.F. Keyser, and R.J. Haug, J. Crust. Growth 249, 477-482 (2003).

46. P. Dawson, Z. Ma, K. Pierz, and E.O. Göbel, AppI. Phuss. Lett. 81, 2349 (2002). 
47. P.W. Fry et al., Phys. Rev. Lett. 84, 733 (2000).

48. D.M. Bruls, J.W.A.M. Vugs, P.M. Koenraad, H.W.M. Salemink, J.H. Wolter, M. Hopkinson, M.S. Strolnick, F. Long, and S.P.A. Gill, Appl. Phys. Lett. 81, 1708 (2002).

49. N. Liu. J. Tersoff, O. Baklenov, A.L. holmes Jr, and C.K. Shih, Phys. Rev. Lett. 84, 334 (2000).

50. O. Wolst, M. Kahl, M. Schardt, S. Malzer, and G.H. Dohler, Phys. E. 17. 554 (2003).

51. A. Lenz et al, Appl. Phys. Lett. 81,5150 (2002).

52. P. Ballet, J.B. Smathers, and G.J. Salamo, Appl. Phys. Lett. 75, 337 (1999).

53. P.B. Joyce, J. Krzyzewski, R. Bell, and S. Jones, Appl. Phys. Lett. 79. 3615 (2001).

54. Q. Gong, R. Nötzel, J. Hamhuis, J. Eijkemans, and H. Wolter, Appl. Phys. Lett. 81, 1887 (2002).

55. This method subtracts the mean value of the nearest neighbour pixels within the filter window: $O(x, y)=I(x, y)-\frac{1}{M \times N} \sum_{i=-N / 2}^{N / 2} \sum_{j=-M / 2}^{M / 2} I(x+i, y+j)$. By choosing a size of about $1 \times 1 \mathrm{~nm}^{2}$ for the filter window, long range fluctuations in the image are suppressed while atomic: details are preserved.

56. A. Rastelli, E. Müller, and H. von Känel, Appl. Phys. Lett. 80, 1438 (2002).

57. M. Stoffel, U. Denker, S. Kar, H. Sigg, and G. Schmidt, Appl. Phys. Lett. 83, 2910 (2003).

58. P.B. Joyce, J. Krzyzewski, R. Bell, A. Joyce, and S. Jones, Phys. Rev B. 58. R15981 (1998).

59. V.M. Ustinov and A.E. Zhukow, Semicond. Sci. Techol. 15, R4 1 (2000).

60. K. Nishi, H. Saito, and S. Sugou, AppI. Phys. Lett. 74, 1111 (1999).

61. E.C. Le Ru, P. Howe, T.S. Jones, and R. Murray, Phus. Status Solidi C. 0, 1221 (2003).

62. H.Y. Liu, M. Hopkinson, C.N. Harrison, M.J. Steer, R. Frith, I.R. Sellers, D.J. Mowbray, and M.S. Skolnick, J. Appl. Phys. 93, 2931 (2003).

63. H.Y. Liu, M.J. Steer, T.J. Badcock, D.J. Mowbray, M.S. Skolnick, P. Navaretti, K.M. Groom, M. Hopkinson, and R.A. Hogg, Appl. Phys. Lett. 86, 143108 (2005).

64. J.M. Ripalda, D. Granados, Y. Gonzalez, A.M. Sanchez, S.I. Molina, and J.M. Garcia, Appl. Phys. Lett. 87, 202108 (2005).

65. S. Frechengues, N. Bertru, V. Drouot, B. Lambert, S. Robinet, S. Loualiche, D. Lacombe, and A. Ponchet, Appl. Phys. Lett. 74, 3356 (1999).

66. P. Caroff, N. Bertru, A. Le Corre, O. Dehaese, T. Rohel, I. Alghoraibi, H. Folliot, and S. Loualiche, Jpn. J. AppI. Phus, 44, L1069 (2005).

67. P. Caroff, C. Paranthoën, C. Platz, O. Dehaese, H. Folliot, N. Bertru, C. Labbé, R. Piron, E. Homeyer, A. Le Corre, and S. Loualiche, Appl. Phys. Lett. 87, 243107 (2005).

68. M.V. Maximow, A.F. Tsatsul'nikov, B.V. Volovik, D.S. Sizov+ Yu.M. Shernyakov. I.N. Kaiander, A.E. Zhukov, A.R. Kovsh, S.S. Mikhrin. V.M. Ustinow, Zh.I. Alferov, R. Heitz, V.A. Shchukin, N.N. Ledentsov, D. Bimberg. Yu.G. Musikhin, and W. Neumann, Phys. Rev. B. 62, 16671 (2000).

69. K. Akahane, N. Yamamoto, and N. Ohtani, Physica E Amsterdam 21, 295 (2004).

70. H.Y. Liu, M.J. Steer, T.J. Badcock, D.J. Mowbray. M.S. Skolnick, F. Suarez, J.S. Ng, M. Hopkinson, and J.P.R. David, J. Appl. Phys. 99, 046104 (2006).

71. R. Songmuang, S. Kiravittaya, and O.G. Schmidt, J. Cryst. Growth 249, 416(2003).

72. WM. McGee, T.J. Krzyzewski, and T.S. Jones, J. Appl. Phys, 99, 043505 (2006).

73. P. Offermans, P.M. Koenraad, J.H. Wolter, K. Pierz, M. Roy+ and P.A. Maksym+ Phys. Rev. B. 72 , $165332(2005)$.

74. G. Costantini, A. Rastelli, C. Manzano, P. Acosta-Diaz, R. Songmuang, G. Katsaros, O.G. Schmidt, and K. Kern, Phys. Rev. Lett. 96, 226106 (2006).

75. P. Offermans, P.M. Koenraad, R. Nötzel, J.H. Wolter, and K. Pierz, AppI. Phys. Lett. 87, 111903 (2005).

76. W. Seifert, D. Hessman, X. Liu, and L. Samuelson. J. Appl. Phys. 75, 1501 (1994).

77. H. Yang, P. Ballet, and G.J. Salamo, J. Appl. Phys. 89, 7871 (2001).

78. B. Wang, F. Zhao, Y. Peng, Z. Jin, Y. Li, and S. Liu, Appl. Phys. Lett. 72, 2433 (1998).

79. P. Disseix, C. Payen. J. Leymarie, and A. Vasson. J. Appl. Phys. 88, 4612 (2000).

80. P.J. Poole, R.L. Williams, J. Lefebvre, and S. Moisa, J. Cryst. Growth 257, 89 (2003).

81. J. He, R. Nötzel, P. Offermans, P.M. Koenraad, O. Gong, G.J. Hamhuis, T.J. Eijlkemans, and J.H. Wolter, Appl. Phys. Lett. 85, 2771 (2004).

82. S. Fafard, Z. Wasilewski, J. McCaffrey+ S. Raymond, and S. Charbonneau, AppI. Phys. Lett. 68, 991 (1996). 
83. H. Marchand, P. Desjardins, S. Guillon, J.-E. Paultre, Z. Bougrioua, R.Y.-F. Yip, and R.A. Masut, Appl. Phys. Lett. 71, 527 (1997).

84. C. Paranthoen, N. Bertru, O. Dehaese, A. Le Corre, S. Loualiche, B. Lambert, and G. Patriarche, Appl. Phys. Lett. 78, 1751 (2001).

85. K. Ozasa, Y. Aoyagi, Y.J. Park, and L. Samuelson, AppI. Phys. Lett. 71,797 (1997).

86. Y. Sakuma, M. Takeguchi, K. Takemoto, S. Hirose, T. Usuki, and N. Yokoyama, J. Vac. Sci. Technol. B. 23, 1741 (2005).

87. N.N. Ledenstov, V.A. Shchukin, M. Grundmann, N. Kirstaedter J. Böhrer, O. Schmidt, D. Bimberg, V.M. Ustinov, A.Yu. Egorow, A.E. Zhukov, P.S. Kop'ev, S.V. Zaitsev, N.Yu. Gordeev Zh.I. Alferow, A.I. Borovkov, A.O. Kosogow, S.S. Ruvimow, P. Werner, U. Gösele, and J. Heydenreich, Phus. Rev. B. $\mathbf{5 4}, 8743(1996)$.

88. Z.R. Wasilewski, S. Farad, and J.P. McCalfrey, J. Cryst. Growth 201, 1131 (1999).

89. T. Raz, D. Ritter, and G. Bahir, Appl. Phys. Lett. 82, 1706 (2003).

90. C. Celebi, J.M. Ulloa, P.M. Koenraad, A. Simon, A. Letoublon, and N. Bertru, Appl. Phys, Lett. 89. $023119(2006)$

91. G. Saint-Girons, G. Patriarche, A. Michon, G. Beaudoin, I. Sagnes, K. Smaali, and M. Troyon, Appl. Phys. Lett. 89.031923(2006).

92. H.R. Gutiérrez, M.A. Cotta J.R.R. Bortoleto, and M.M.G. de Carvalho, J. AppI. Phys. 92. 7523 (2002).

93. J.-S. Lee, H.-W. Ren, S. Sugou, and Y. Masumoto, J. Appl. Phys. 84, 6686 (1998).

94. J.M. Ulloa, C. Celebi, P.M. Koenraad, A. Simon, E. Gapihan, A. Letoublon, N. Bertru+ J. Drouzas, D.J. Mowbray, M.J. Steer and M. Hopkinson. J. Appl. Phys, 101, 081707 (2007).

95. J.M. Ulloa, I.W.D. Drouzas, P.M. Koenraad, D.J. Mowbray, M.J. Steer, H.Y. Liu and M. Hopkinson. Appl. Phys. Lett. 90, 213105 (2007).

96 J.M. Ulloa, P.M. Koenraad, E. Gapihan, A. Létoublon and N. Bertru, Appl. Phys. Lett. 91, 073106 (2007). 